\title{
Congenital Anomalies of the Kidney and the Urinary Tract (CAKUT)
}

\author{
Maria M. Rodriguez \\ Holtz Children's Hospital Department of Pathology, Pediatric Pathology, University of \\ Miami, Coral Gables, FL, USA
}

This article reviews the majority of Congenital Anomalies of the Kidney and Urinary Tract (CAKUT) with emphasis in Pediatric Pathology describing and illustrating lesions as varied as ureteral duplications, ureteropelvic junction obstruction, horseshoe kidney, posterior urethral valve and prune belly syndrome, obstructive renal dysplasia, nonmotile ciliopathies and several syndromes associated with renal malformations (Meckel-Joubert, short rib, Bardet-Biedl, asplenia/polysplenia, hereditary renal adysplasia, Zellweger, trisomies, VACTER-L, Potter, caudal dysplasia, and sirenomelia), as well as ADPK, and ARPK.

The purpose of this review is not only to describe the congenital renal anomalies, but also to analyze the more recent therapeutic interventions that may modify the natural history of some of these severe conditions.

\section{INTRODUCTION}

"Give a man a fish and he eats for a day, teach him to fish and he can feed himselffor life."

Dr. Enid Gilbert-Barness is an extremely distinguished and well-known pediatric pathologist that has educated generations of medical students, residents, and fellows, written numerous books, chapters, and articles in the field of embryo-fetal and pediatric pathology during her long career and is now relinquishing her position as the Editor-in-Chief of Fetal and Pediatric Pathology.

I am very humbled to accept the invitation by the publisher to contribute a manuscript to the festschrift in her honor.

If one tries to define Dr. Gilbert-Barness' areas of interest in pediatric pathology, one will realize that she has written about almost everything within the field. One of her most notable contributions has been as the editor of Potter's Pathology of the Fetus, Infant and Child. This book was originally edited by Dr. Edith Potter, who is considered "the mother of perinatal pathology," and is recognized as the first pathologist to describe the mechanical deformities associated with oligohydramnios sequence due

Received 26 August 2014; Revised 26 August 2014; accepted 26 August 2014.

This is an open-access article distributed under the terms of the CC-BY-NC-ND 3.0 License which permits users to download and share the article for non-commercial purposes, so long as the article is reproduced in the whole without changes, and provided the original source is credited. Address correspondence to Dr Maria Matilde Rodriguez, MD, University of Miami, Pathology, Holtz Children's Hospital, 1611 NW 12 Avenue, Department of Pathology, Miami, Fl 33136, USA. E-mail:mmrod@miami.edu 
to bilateral renal agenesis, also known as Potter syndrome. It is for that reason that I have decided to write about congenital anomalies of the kidney and urinary tract.

The spectrum of congenital anomalies of the kidney and urinary tract is extremely broad and ranges from mild, asymptomatic malformations such as a double ureter or minimal ureteral pelvic obstructions to severe, life-threatening pathologies like bilateral renal agenesis or renal dysplasia. Several of these renal abnormalities are part of a syndrome or sequence that can be confirmed and sometimes treated by a multidisciplinary approach including fetal ultrasonography and vesico-amniotic shunt placement to relieve obstruction while in the fetal period, or by other imaging modalities, molecular analysis, and pathologic examination after birth.

The purpose of this article is to illustrate the majority of lesions involving the renal system, including the pathologic alterations associated with syndromes, and to analyze the more recent therapeutic interventions that may modify the natural history of some of these severe conditions.

\section{Double Ureter or Ureteral Duplication}

Ureteral duplication may be incomplete or complete. Incomplete duplication is also known as a bifid collecting system. If there are two separate pyelocalyceal systems and they join at the ureteropelvic junction (UPJ), it is considered a bifid pelvis; if there are two separate ureters at the proximal aspect and they join at any point below the UPJ, but before entering into the bladder, the patient is considered to have bifid ureters. Complete ureteral duplication is when there are two separate ureters that continue and enter the urinary bladder [1].

The majority of cases are asymptomatic in adults; however, in children the risk of renal infection is increased 20 -fold. Atwell et al. studied 30 children with a duplex urinary tract along with their parents and siblings [2]. It was found that if an incomplete duplication (bifid pelvicalyceal system) was included as a variant of a duplex system, 20 families (66\%) had affected first-degree relatives. Since usually only one of the parents was affected, the mode of inheritance was established as autosomal dominant.

The so-called conjoint ureter is more common than complete ureteral duplication. If one of the two parts ends blindly, it is named a congenital ureteral diverticulum [3] and double ureter is more common in girls than in boys in a ratio of 6:1. Girls may have urinary incontinence when the ectopic ureter opens in the urethra distal to the sphincter. A duplex system can be associated with other renal complications such as obstruction, reflux, and infection. If the obstruction is maintained for some time, the kidney can become hydronephrotic. When the infection becomes persistent, it can also lead to a severe chronic pyelonephritis, which ultimately produces chronic renal disease. A relatively recent treatment modality for pediatric urologic anomalies is laparoscopic polar nephrectomy with ureteropyeloanastomosis [4]. The procedure involves a surgical anastomosis of the upper pole ureter to the renal pelvis draining the lower pole, with prior insertion of a double $J$ stent. The laparoscopic procedure is minimally invasive with rapid postoperative recovery and good long-term results.

It is uncommon to receive a nephrectomy specimen with a double ureter in surgical pathology since the majority of these kidneys are corrected by ureteropyeloanastomosis, but this anomaly can be associated with other more serious pathologic conditions such as Wilms' tumors or renal dysplasia that will require nephrectomy. The kidney depicted in Figure 1 is a nephrectomy secondary to Wilms tumor. Coincidentally, that kidney also had ureteral duplication.

\section{Ureteropelvic Junction (UPJ) Obstruction}

This is one of the most common surgical specimens received in Pathology from the pediatric urologists. UPJ obstruction has an incidence of 1 in 1000-1500 newborns and 


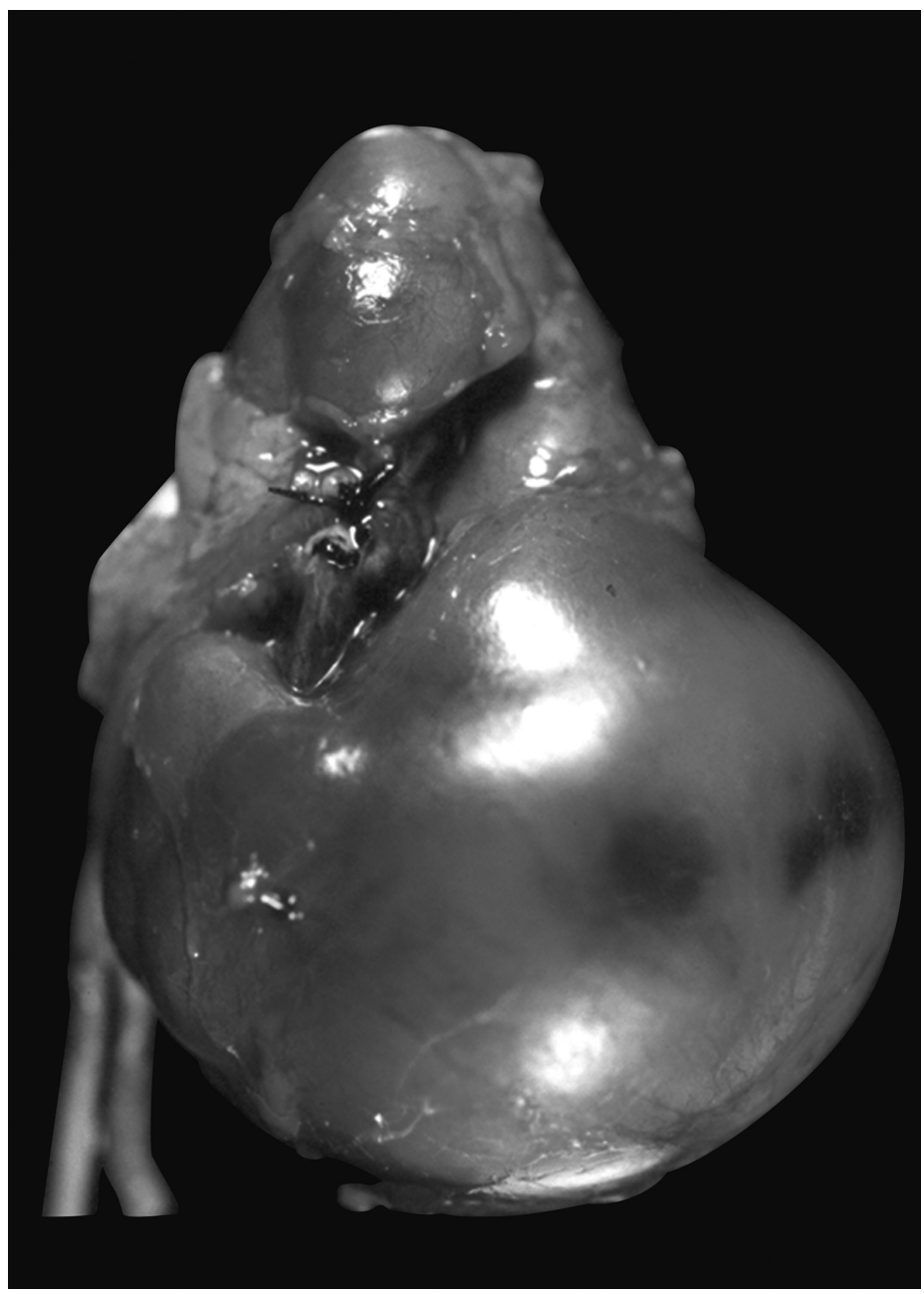

Figure 1. The nephrectomy was performed because of a Wilms tumor. However, the kidney had a double ureter.

is the most common cause of antenatal hydronephrosis [5]. The pathophysiology of UPJ obstruction seems to be multifactorial and there are several articles supporting different theories that can be summarized as follows:

- Obliteration-recanalization theory by Ruano-Gil and Tejedo-Mateu [6]: Human embryos between 5 to $55 \mathrm{~mm}$ were studied and found to have a temporary obliteration of the ureteric duct (future ureter) followed by recanalization. That theory has not been widely accepted as it is believed that even though the ureter is collapsed, it is still patent.

- Extrinsic obstruction by abnormal blood vessels: Fetal external ureteral compression can produce incomplete muscularization of the ureter and narrow its lumen.

- Id2 haploinsufficiency and hydronephrosis: Both homozygous and heterozygous Id2 mutant mice developed hydronephrosis due to UPJ obstruction. The hydronephrosis was congenital, more common in males and unilateral (right kidney that is positioned higher than the left as in humans). These authors found no abnormal vessels or any other type of external compression of the UPJ [7].

- Other authors postulate that congenital UPJ obstruction is associated with smooth muscle cell apoptosis and defective neural development. They studied the UPJ's 


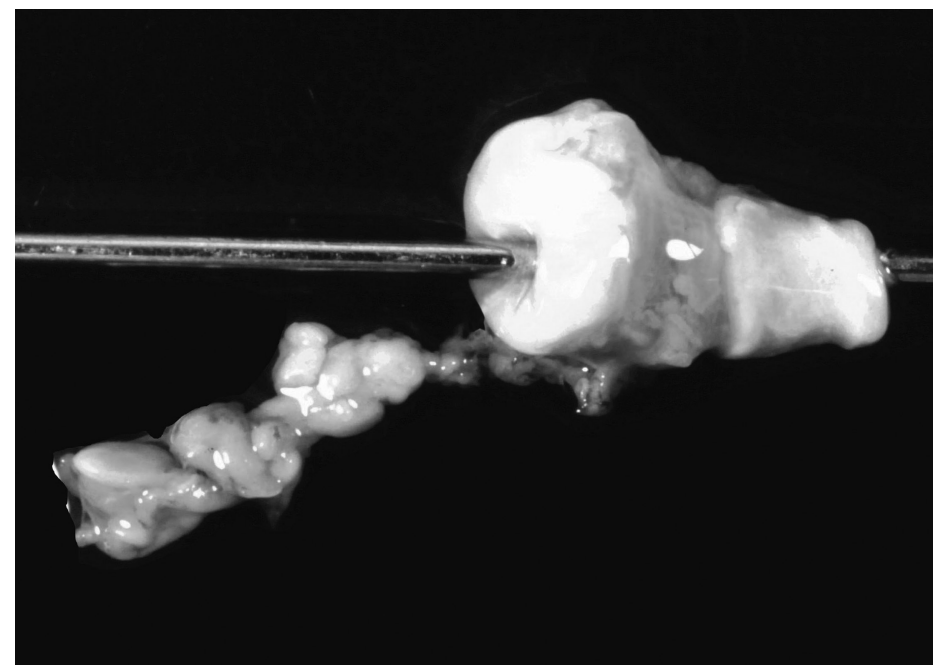

Figure 2. The ureteropelvic junction (UPJ) was probed after stretching the kink in the lumen and it was patent, but stenotic. The patient had been symptomatic for years and required surgery.

from 23 patients that underwent pyeloplasty for correction of obstruction and compared them to 25 UPJ's from autopsy material taken from cadavers with no evidence of obstruction. The sections were stained with neurofilament (NF) protein antibody and the positively stained fibers were markedly reduced in sections from patients with UPJ obstruction when compared to controls. Moreover, there was an increase in smooth muscle cell apoptosis in UPJ obstruction specimens [8].

Since the practical viewpoint for the pathologist receiving a portion of ureteropelvic junction (UPJ), the main problem resides in the luminal narrowing that can be explored by probing it (Figure 2). There are some instances in which the lumen is not probe patent and others in which there is a kink leading to stenosis. The proximal portion of the pelvis is usually dilated. After probing the lumen, it is advisable to sample cross sections of the renal pelvis, the stenotic area, and distal ureter if available. There are many causes of UPJ obstruction or stenosis including insertion anomalies of the ureters, ureteral muscular hypertrophy, peripelvicalyceal fibrosis, and abnormal blood vessels crossing over the ureter or renal pelvis. Long standing obstruction may lead to pyelonephritis, hydronephrosis, and renal failure.

\section{Horseshoe Kidney or Renal Fusion}

The majority of cases are fused at the lower pole. Fusion is twice as common in males. It is usually associated with a narrow pelvis as seen in trisomy 18. Most fused kidneys are positioned lower than normal. Sometimes the horseshoe kidneys are associated with UPJ obstruction and children can present with urinary tract infections, abdominal mass, and hematuria. One alternative treatment is transperitoneal laparoscopic pyeloplasty that allows a better exploration of the pyelocalyceal system and detection of anatomical anomalies such as crossing vessels that are more commonly found in horseshoe kidneys [9].

\section{Posterior Urethral Valves and Prune Belly Syndrome (PBS)}

Posterior urethral valves are considered the most common cause of lower urinary tract obstruction in male infants and fetuses [10]. PBS affects 3.8 per 100000 live male births. These fetuses have a massively distended abdomen due to megacystis. The urinary 

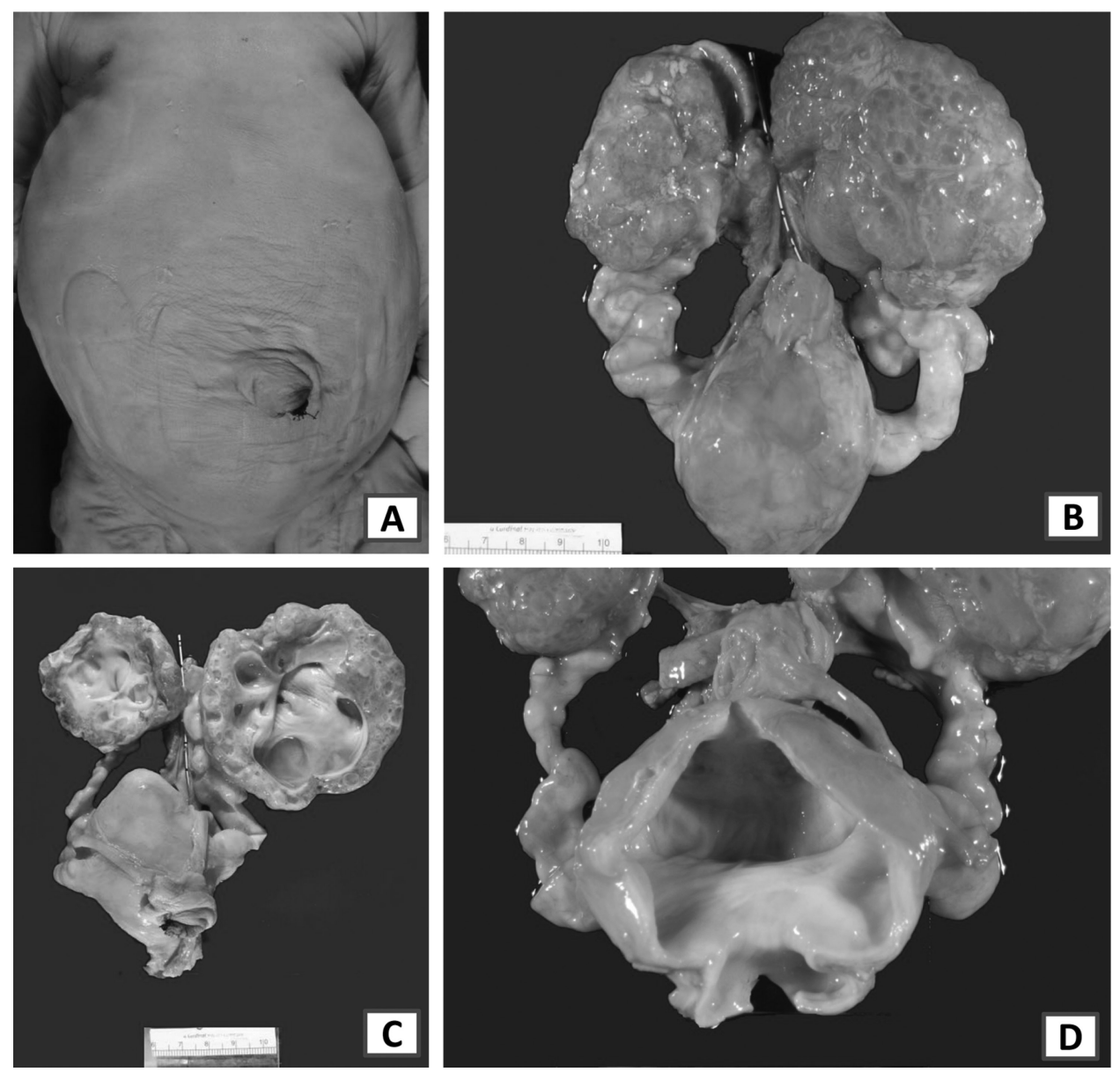

Figure 3. (A) After urine was drained from the urinary bladder (UB), the neonate's skin wrinkled like a prune. Observe the small chest in contrast to the large abdomen. The pulmonary hypoplasia is secondary to a combination of elevated diaphragm and oligohydramnios. (B) Both kidneys are multicystic and dysplastic, the ureters are tortuous and dilated and the UB is thick and dilated. (C) The kidneys were bivalved and were hydronephrotic. (D) The urinary bladder demonstrates posterior urethral valves.

bladder could be drained by vesico-amniotic shunt in utero or by vesicocentesis after birth. After drainage, the abdomen wrinkles and acquires the appearance of a prune, hence the name prune-belly syndrome (Figure 3).

Since fetal urine is an important component of amniotic fluid, posterior urethral valves will produce oligohydramnios with a distended urinary bladder, bilateral hydronephrosis, and obstructive renal dysplasia. In many instances, the urinary bladder has a hypertrophic muscular wall and is massively dilated by urine accumulation. The subsequent pressure over the abdomen produces atrophy of the abdominal muscles and leads to an abdomen covered only by skin and peritoneum or by an extremely atrophic muscular layer. Survival of these children depends on the degree of pulmonary hypoplasia which usually results in severe respiratory insufficiency. They also have undescended testis and intestinal malrotation secondary to the distended urinary bladder that does not allow for normal bowel positioning or testicular descent. If the obstruction is not treated in utero, these children develop oligohydramnios sequence with low-set ears, flat nose, folds under the eyes, varus deformities of feet, etc. 
Prune belly syndrome is associated with a genomic $\operatorname{HNF} 1 \beta$ (hepatocyte nuclear factor) mutation in 3\% of cases. Granberg et al. studied 32 DNA samples from individuals affected with PBS (30 males and 2 females). One heterozygous mutation was detected in exon 1 of the $\mathrm{HNF}_{1} \beta$ gene, yielding a missense mutation encoding glycine in lieu of valine at the 61 position [11]. The same mutation has been reported in two patients without PBS but one with VACTERL association and the other with the combination of a mullerian anomaly and a solitary kidney [12]. According to these authors, the mutation has been found in 9 of 108 women with uterine and renal anomalies (8\%), but it has never been found in women with an isolated uterine anomaly. The uterine malformations vary from bicornuate to absent, or hypoplastic uterus. The most common renal anomaly is multiple renal cysts, but there are cases of single kidney or endstage renal disease of unknown etiology.

More recently, the fetal lower urinary tract obstruction (LUTO) is being managed by placing a vesico-amniotic shunt endoscopically that allows fetal urine to drain in the amniotic sac and diminishes the severity of the oligohydramnios and all the other consequences including pulmonary hypoplasia [13]. However, one of the long-term complications of vesico-amniotic shunt placement can be bilateral obstructive renal dysplasia associated with chronic renal failure in infants that survive the neonatal period despite the positive outcome of improved pulmonary development.

\section{Obstructive Versus Nonobstructive Renal Dysplasia}

Currently, it is well recognized that there are two different types of renal dysplasias: obstructive and nonobstructive. In many instances of obstructive renal dysplasia (ORD), a megacystis (massively distended urinary bladder) can be observed, even by fetal ultrasound; however, there are other cases in which the obstructive component is not as obvious and only a careful examination will detect a stenotic area, a hydroureter, or a dilated renal pelvis. Histologically, when kidneys affected by ORD have developed hydronephrosis, they usually exhibit a compressed medulla due to the hydrostatic pressure produced by the accumulated urine within the pelvis. The renal cortex tends to have less fibrosis and more glomeruli while the medulla is fibrotic and dysplastic (Figure 4); however, the histology may vary depending on the timing of the intrauterine obstruction.

We have studied a series of patients with multicystic dysplastic kidneys and found that approximately $60 \%$ of kidneys affected by renal dysplasia have an obstructive component. Among our patients with ORD, five children had prune belly syndrome, two had unilateral renal agenesis, hydroureters, and hypoplastic urinary bladder, and two had VACTER association with hydronephrosis [14]. We stained the histological sections by immunohistochemistry for insulin growth factor (IGF2), paired box gene 2 (PAX2), transforming growth factor $\beta$ (TGF- $\beta$ ), and B-cell lymphoma-2 (BCL-2), and compared them to fetuses and children with multicystic dysplastic kidneys with no evidence of obstruction and some of them with syndromes associated with renal dysplasia including: Meckel-Gruber syndrome, trisomy 18, asplenia, and polysplenia. There was no immunoreactivity for TGF- $\beta$ and IGF2 in sections from ORD, while the antibodies were expressed in nonobstructive dysplastic kidneys. Since TGF is involved in the mesenchymal-to epithelial transformation, we postulate that the mechanism by which the kidneys develop dysplastic changes in nonobstructive dysplasia is mediated by TGF, while the obstructive type seems to be derived from increased hydrostatic pressure in the renal pelvis. In our study, there were no statistically significant differences in the pattern of staining of PAX2 and BCL-2 in obstructive versus nonobstructive renal dysplasia.

Insulin growth factor (IGF) has been proven to be overexpressed in multicystic renal dysplasia [15]; however, these authors did not differentiate between obstructive 

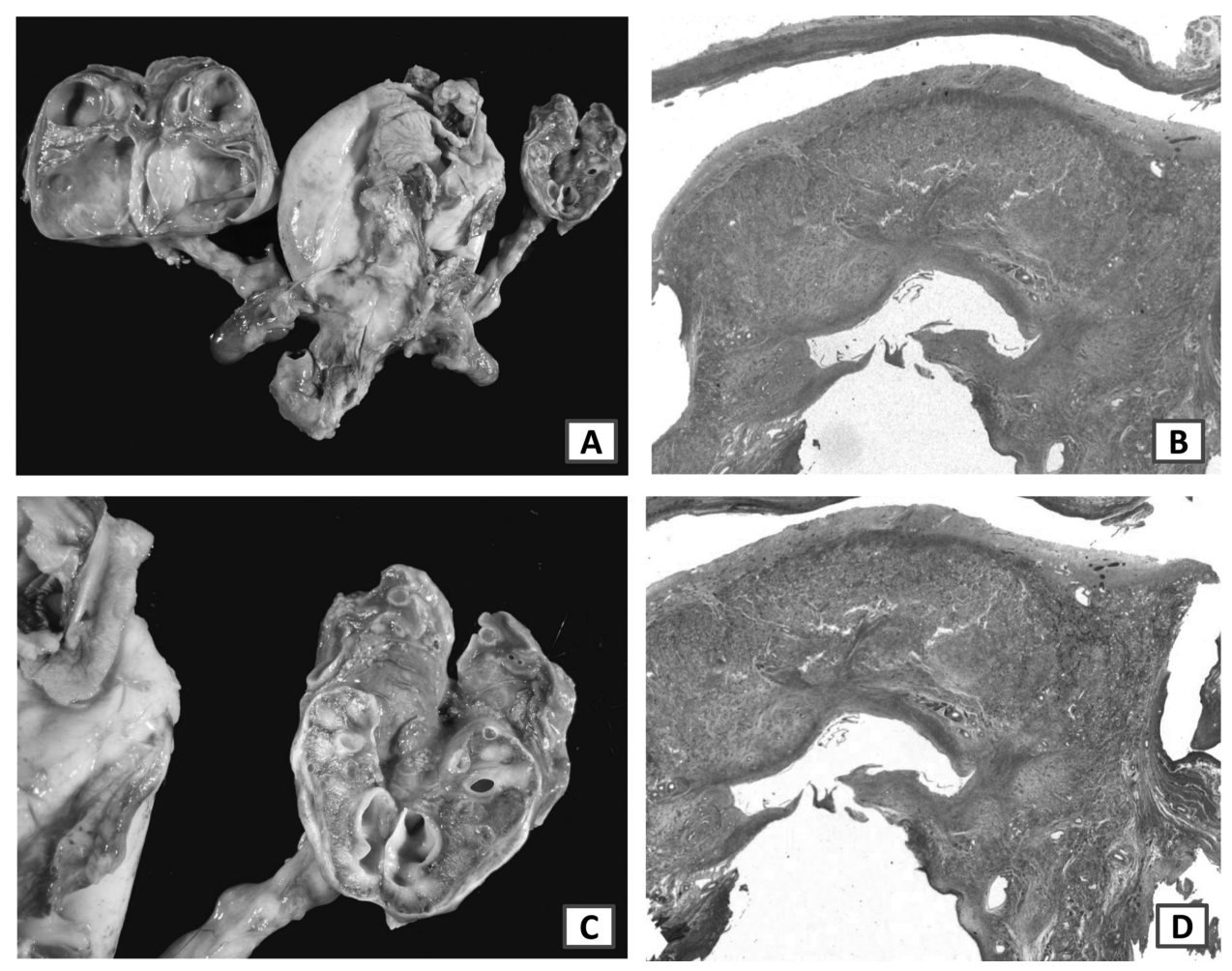

Figure 4. Composite photograph of obstructive renal dysplasia with hydronephrosis. (A) Both kidneys were dissected with the ureters still attached to the UB and then bivalved to demonstrate the hydronephrosis. (B) Whole-mounted section from the kidney was stained with H\&E. The cortex is relatively well preserved while the medulla is more compressed by the hydrostatic pressure of the urine in the renal pelvis. (C) Close-up view of the kidney affected by hydronephrosis. (D) Wholemounted section from the kidney was stained with trichrome. Observe the better preserved renal cortex compared to the fibrotic medulla in blue.

and nonobstructive types. Nonobstructive can be syndromic and nonsyndromic (Figure 5).

In order to better understand the etiology and pathogenesis of renal dysplasia, many efforts have been made to further classify them as syndromic and nonsyndromic and to find the gene or genes involved in the development of the disease. A very comprehensive review article by Dr. Yosypiv delineates evidence that $2 \%$ of the congenital anomalies of the kidney and urinary tract, even if nonsyndromic, are linked to genes [16]. So far, the following genes have been reported: hepatocyte nuclear factor- $1 \beta$ (HNF1 $\beta$ ), paired box gene 2 (Pax2), uromodulin (UMOD), and eyes absent homolog 1 (Eyal). They have been detected in $2 \%$ of patients with nonsyndromic congenital anomalies of the kidney and urinary tract (CAKUT).

A large study by Saisawat et al. [17] applied massive parallel exon resequencing of 30 candidate genes in pooled DNA from 40 patients with CAKUT and identified seven novel mutations in four genes: RET, BMP4, FRAS1, and FREM2. All of these mutations were absent in healthy controls.

\section{Nonmotile Ciliopathies and Congenital Renal Diseases}

During the last decade, a number of syndromes have been linked to primary ciliary dysfunction. The list continues to grow, but there are some that are relatively 

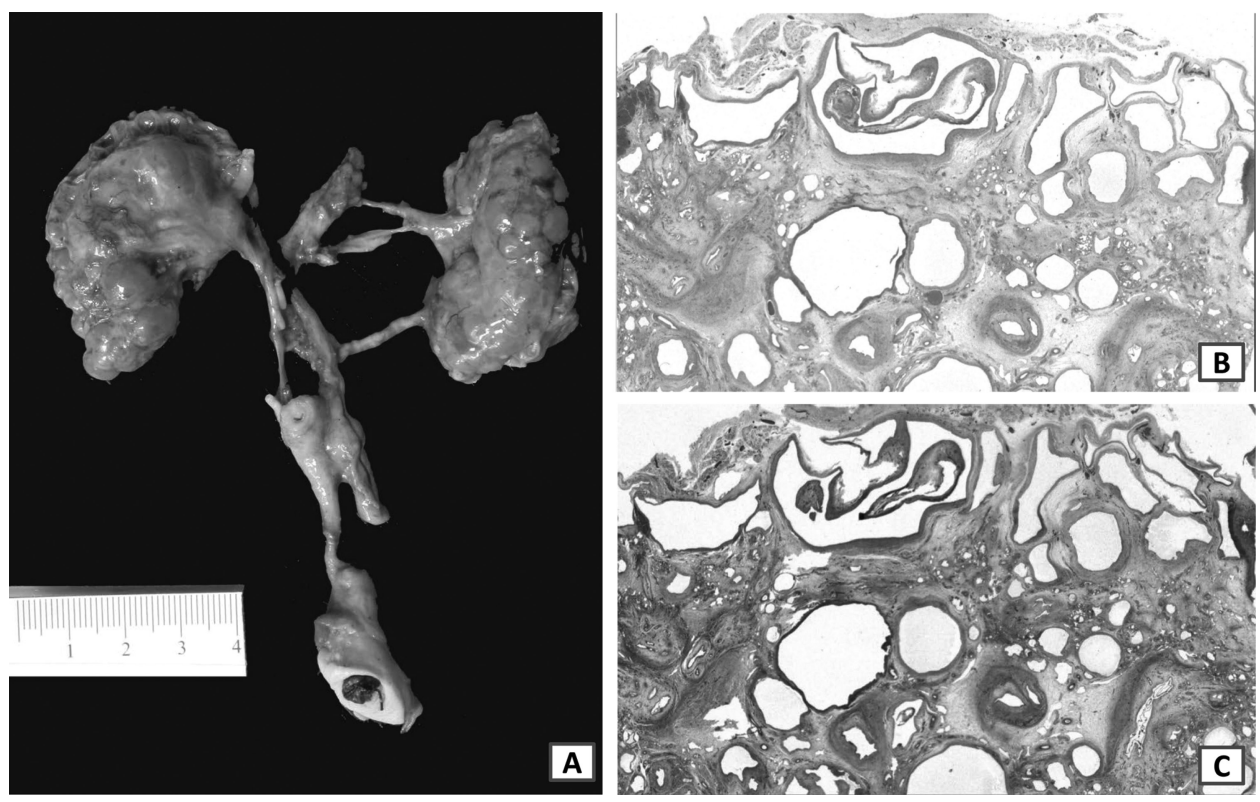

Figure 5. Gross and photomicrographs from nonobstructive renal dysplasia. (A) The kidneys were left attached to the aorta by the renal arteries. The ureters are of normal caliber. The UB is small because the kidneys were producing a very small amount of urine. (B) H\&E-stained whole-mounted section from the kidney. The parenchyma is disorganized and there are numerous cysts surrounded by concentric fibrosis. (C) Trichrome-stained whole-mounted section from the kidney. There is extensive fibrosis throughout this kidney (stained in blue).

well characterized: Bardet-Biedl syndrome, nephronophthisis, Alström syndrome, Meckel-Gruber, Joubert, Jeune asphyxiating thoracic dystrophy, short rib with and without polydactyly, and Ellis van Creveld.

There are several types of cilia in the human body and their functions differ one from the other. The embryonic node is a motile monocilium involved with laterality and plays a role in asplenia and polysplenia. Multiple motile cilia can be found in respiratory epithelial cells, brain ependymal cells, and epithelial cells in fallopian tubes, and sperm have a single elongated motile cilium. However, in this section we will refer to tissues that are endowed with nonmotile monocilia such as renal tubular, bile duct, pancreatic epithelia, osteocytes, and chondrocytes [18]. There are several common features in syndromes associated with nonmotile ciliopathies: renal and hepatobiliary cysts, laterality defect (situs inversus), polydactyly, agenesis of corpus callosum, cognitive impairment, retinal degeneration, posterior fossa defects or encephalocele, skeletal anomalies, and obesity.

The most common syndromes related to renal dysplasia, according to my experience, are Meckel-Gruber syndrome, Joubert syndrome, short rib syndrome, asplenia/polysplenia syndrome, and VACTER-L.

\section{Meckel-Gruber Syndrome}

This syndrome was first described by Johann Friedrich Meckel the Younger in 1822. He described two sibling neonates, a boy and a girl who had the combination of microcephaly with occipital encephalocele, cleft palate, polydactyly, and large cystic kidneys. According to Meckel, "In the boy, the testes were near the lower pole of the kidneys and the external genitalia were more female-like." Meckel mentioned that he suspected that the infants were an example of heredity (Erblichkeit) because he had 

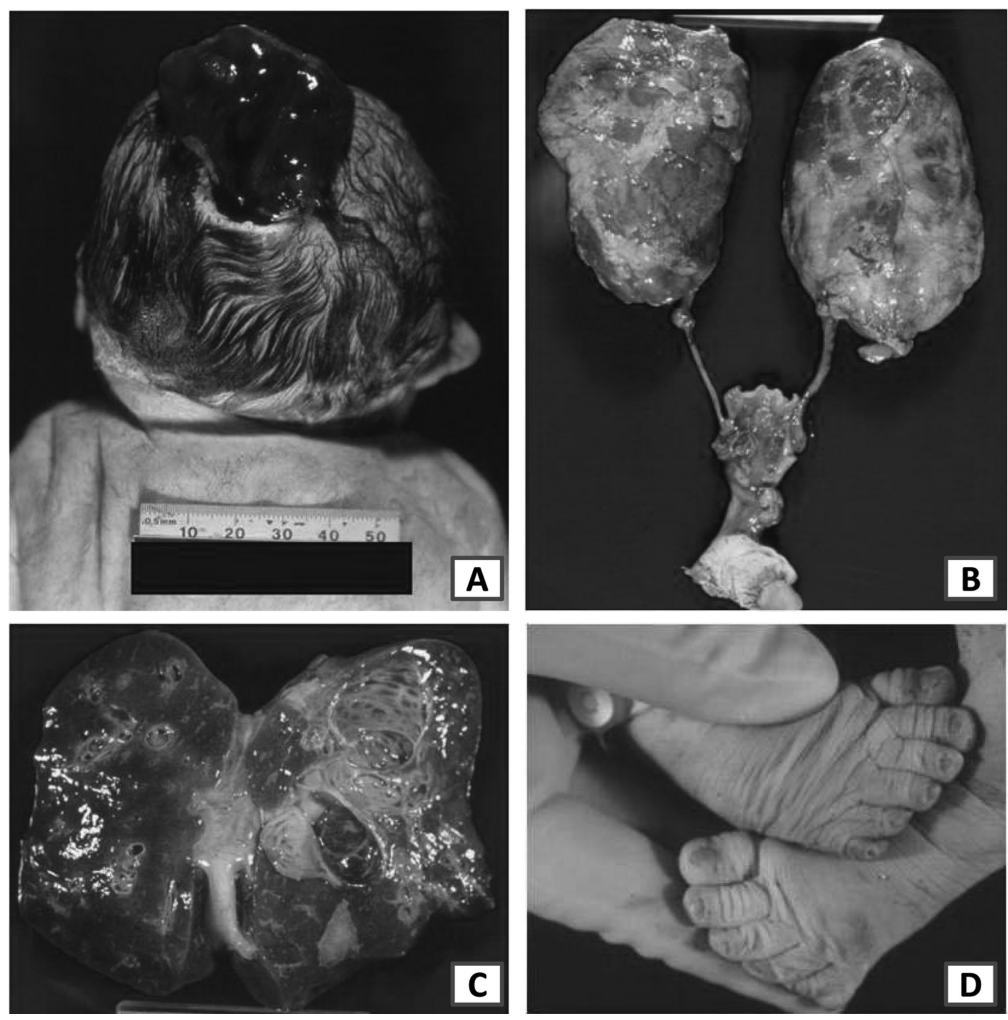

Figure 6. Composite photograph of a patient with Meckel syndrome. (A)Posterior view of a child with an occipital encephalocele. The ears are low set. (B)Both kidneys are dysplastic and the ureters as well as the urinary bladder are small as seen in syndromic (nonobstructive) renal dysplasia. (C) Cut section throughout the liver demonstrates areas of fibrosis mainly at the right lobe. Histologically, the liver disclosed congenital hepatic fibrosis. (D) Polydactyly of both feet. The hands were normal.

seen other instances of anomalies within given families [19]. More than a century after, Gruber reported a few similar cases he had encountered and added a few more from the literature for a total of 16 cases. Gruber named the disease dysencephalia splanchnocystica and mentioned that it was genetic because many of them were among siblings [20]. We have autopsied three siblings with Meckel-Gruber syndrome. They include a full term boy born at 40 weeks, a male fetus delivered at 19 weeks, and a fullterm girl born at 41 weeks. All three cases had multicystic dysplastic kidneys, polydactyly of all extremities, and microcephaly. Both fullterm infants had congenital hepatic fibrosis. The fullterm male had anencephaly and the other two each had an occipital encephalocele (Figure 6).

Currently, we know that its mode of inheritance is autosomal recessive and there are many types according to the cytogenetic location (Table 1). Meckel syndrome is usually lethal in early infancy due to the severity of renal and central nervous system (CNS) malformations. It is characterized by the combination of renal cystic dysplasia, occipital encephalocele, cleft lip and/or cleft palate, polydactyly, oligohydramnios, congenital hepatic fibrosis, and ambiguous genitalia. However, there are other CNS anomalies that have been described in these children including sloping forehead, hydrocephalus, prosencephaly and/or arhinencephaly, absence of lateral ventricles, craniorachischisis, and anomalies of the corpus callosum. 
Table 1. Meckel syndrome types according to On-line Mendelian Inheritance in Man (OMIM) and cytogenetic locations.

\begin{tabular}{lcc}
\hline Meckel syndrome type & OMIM \# & Cytogenetic location \\
\hline MKS1 & 249000 & $17 \mathrm{q} 22$ \\
MKS2 & 603194 & $17 \mathrm{q} 12.2$ \\
MKS3 & 607361 & $8 \mathrm{q} 22.1$ \\
MKS4 & 611134 & $12 \mathrm{q} 21.32$ \\
MKS5 & 611561 & $16 \mathrm{q} 12.2$ \\
MKS6 & 612284 & $4 \mathrm{p} 15.32$ \\
MKS7 & 260710 & $3 \mathrm{q} 22.1$ \\
MKS8 & 613885 & $12 \mathrm{q} 24.31$ \\
\hline
\end{tabular}

\section{Joubert Syndrome and Related Disorders}

This syndrome is an uncommon autosomal recessive condition characterized by hypoplasia of the cerebellar vermis (demonstrated by magnetic resonance imaging as the "molar tooth sign"), intellectual disability, retinal dystrophy, ocular coloboma, rotatory nystagmus, polydactyly, cystic renal dysplasia, and congenital hepatic fibrosis. Its neurologic manifestations usually come to medical attention in infancy. The syndrome was initially described in a French-Canadian family with four affected siblings by pediatric neurologists in Montreal more than 40 years ago [21]. One of the original children had an occipital meningoencephalocele that was removed at birth.

Even though renal disease was not mentioned by Dr. Marie Joubert and her group in their original description, the prevalence of kidney problems in Joubert syndrome and related disorders (JSRD) has been estimated as $30 \%$. When children are followed long term, the percent is even higher. The two different forms of renal disease that have been described in Joubert are cystic renal dysplasia and nephronophthisis (tubulointerstitial nephritis and cysts at the cortico-medullary junction). Clinically, these children present with polydypsia, polyuria, anemia, growth failure, elevated creatinine, and later develop renal failure.

Nowadays it is known that Joubert syndrome is due to ciliary disorders and there is some overlap in clinical presentations with Meckel and Bardet-Biedl syndromes (occipital meningoencephalocele, congenital hepatic fibrosis, obesity, ambiguous genitalia, etc.) So far, the molecular genetics of JSRD includes eight mutations in the ciliary/basal body genes: INPPFE, AH11, NPHP1, CEP290, TMEM67/MKS3, RPGR1P1L, $A R L 13 B$, and CC2D2A [22]. At a molecular level, it has been demonstrated that Joubert and Meckel syndromes share at least one gene mutation (TMEM67/MKS3) and it seems logic to infer that in the future more common mutations will be found.

We performed an autopsy on a child with Joubert syndrome that had a massively distended abdomen due to nephromegaly with cystic renal dysplasia, absent cerebellar vermis, and congenital hepatic fibrosis. The child died 2 days after birth of respiratory insufficiency (Figure 7). The history did not reveal any other family members with these or similar manifestations.

\section{Short Rib Syndrome}

Short rib syndrome (SRS) is one of the lethal osteochondrodysplasias and has been traditionally divided into four types (I-Saldino-Noonan, II-Majewski, III-VermaNaumoff, and IV-Beemer-Langer). These autosomal recessive, skeletal ciliopathies are characterized by a narrow thorax due to short ribs, cleft lip, and/or cleft palate, cystic renal dysplasia, congenital hepatic fibrosis, pancreatic cysts, ocular and cerebral anomalies, as well as abnormal genitalia. Polydactyly is variably present. The histology of the kidneys and liver is undistinguishable from the findings in patients with 

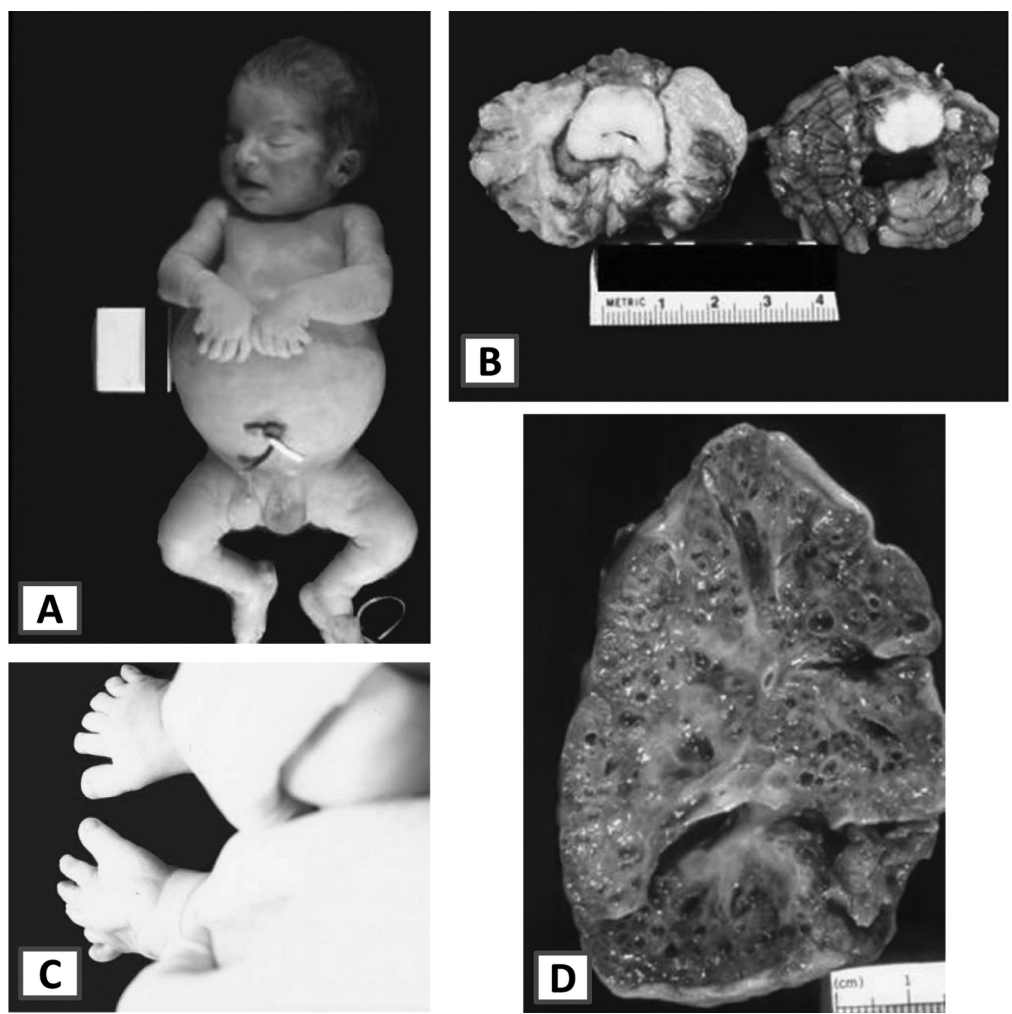

Figure 7. Newborn with Joubert syndrome. (A) The neonate had a parrot-beak nose, slightly lowset ears, a distended abdomen and bilateral post-axial polydactyly. (B) The cerebellar vermis was absent. (C) Both feet also demonstrated postaxial polydactyly. (D) The kidneys lost their reniform shape, were large and contained multiple round cysts.

Meckel and Joubert syndromes. We reported a newborn that also had pancreatic cysts, bilateral cystic renal dysplasia, congenital hepatic fibrosis, atrophic optic chiasm, microphthalmus, three accessory spleens, but no situs inversus (Figure 8). The affected child died in the neonatal period due to pulmonary hypoplasia leading to respiratory insufficiency [23].

Through Sanger sequencing analysis of NIMA-related kinase 1 gene (NEK1), nucleotide changes were identified in three families with children affected with the SRS Majewski type [24]. Affected individuals had a narrow thorax, hypoplastic lungs, polysyndactyly, disproportionate dwarfism, and cleft lip and palate. One of the families had ventricular septal defects and cystic kidneys. Radiologic studies confirmed shortened and horizontal ribs, squared scapulae, elevated clavicles with lateral kinking, normal spine and pelvis, and shortening of the bones of all extremities. Davis et al demonstrated that mutations in TTC21B, which encodes the retrograde intraflagellar transport protein IFT139, produces nephronophthisis and Jeune asphyxiating thoracic dystrophy [25]. However, they believe that this gene mutation may be responsible for approximately $5 \%$ of cases of ciliopathies. These mutations can also modify penetrance and severity of disease.

\section{Bardet-Biedl Syndrome}

The cardinal manifestations of Bardet-Biedl syndrome are retinitis pigmentosa, obesity, renal dysplasia, polydactyly, learning disability, and hypogenitalism [26]. The 


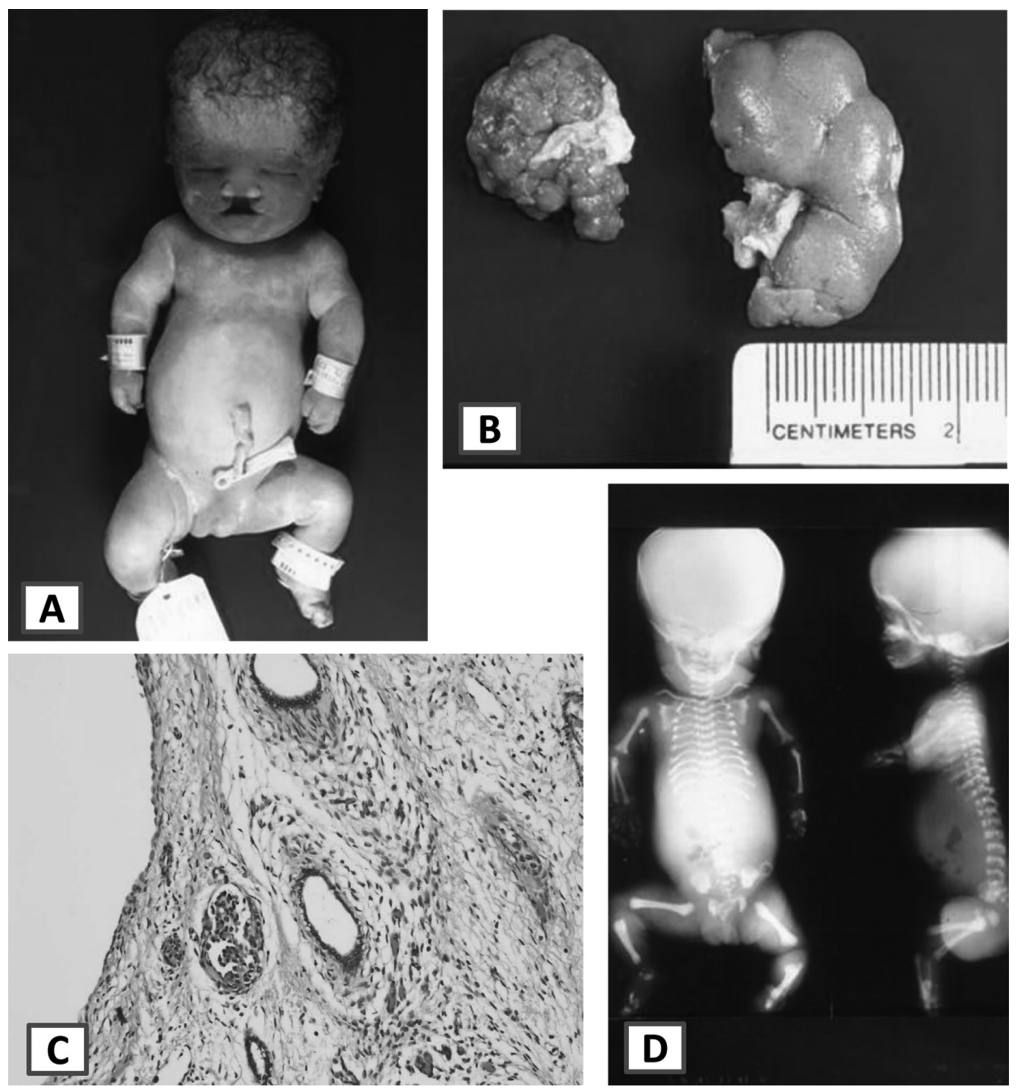

Figure 8. Newborn with short-rib syndrome who died during the first day of life due to respiratory insufficiency. (A) The baby had a large cranium with large fontanelles, a median cleft lip and palate, bilateral anophthalmia, a very small thorax and a protuberant abdomen. (B) The left kidney weighed less than half the weight of the right and both had cystic renal dysplasia. (C) The renal parenchyma is disorganized with concentric fibrosis around two dilated tubules. There is a single glomerulus in the photo next to a large, cystically-dilated space to the left. (D) Antero-posterior and lateral X-rays demonstrate large cranium, short ribs, hypoplastic ilia, and short, symmetrical extremities.

diagnosis may be confirmed later as a young adult if the patient presents in childhood only with truncal obesity and learning disabilities, but has normal vision and lacks polydactyly or syndactyly and hypogonadism. However, as patients age, visual and renal problems become more severe and virtually $100 \%$ of individuals with Bardet-Biedl syndrome will develop poor vision. The possibility of renal insufficiency increases with age. It is inherited in an autosomal recessive manner. Interfamilial and intrafamilial phenotypic variability exist. Prenatal diagnosis in affected families can be made by fetal ultrasound examination with detection of polydactyly and cysts in the kidneys. Once the affected gene has been detected in a family, it is possible to look for the known gene. At the present time, 18 genes have been associated with Bardet-Biedl syndrome: BBS1, BBS2, ARL6 (BBS3), BBS4, BBS5, MKKS (BBS6), BBS7, TTC8 (BBS8), BBS9, BBS10, TRIM32 (BBS11), BBS12, MKS1 (BBS13), CEP290 (BBS14), WDPCP (BBS15), SDCCAG8 (BBS16), LTZFL1 (BBS17), and BBIP1 (BBS18). Histologically, the kidneys show extensive replacement of parenchyma by round cysts lined by flat to cuboidal epithelium [27]. The glomeruli are preserved. Persistent fetal lobulations have been described suggesting a defect in renal maturation. 


\section{Renal-Hepatic-Pancreatic Dysplasia (RHPD) and Asplenia/Polysplenia}

There are several reports in the medical literature describing children with this autosomal recessive syndrome seen in ciliopathies; some of them also have situs inversus with polysplenia or asplenia. The first report consisting of two siblings with renal-hepaticpancreatic dysplasia and asplenia seems to be by Ivemark et al in 1959 [28]. There have been other case reports: one by Crawfurd that described two siblings, a boy and a girl with enlarged, multicystic kidneys, cystic pancreas, and portal fibrosis. They also had either hypoplastic or absent spleen and cardiac anomalies [29]. The largest series so far was written by the late Dr. Jay Bernstein and collaborators in 1987 and it was composed of five infants: three of them died in the neonatal period and the other two died in infancy. They all had cystic renal dysplasia and liver abnormalities with elongated bile ducts surrounded by fibrosis. Subsequent liver biopsies in the longer surviving patients demonstrated paucity of bile ducts and later biliary "dysgenesis" [30]. They concluded that cases of renal-hepatic-pancreatic dysplasia do not necessarily constitute a homogeneous group.

At the molecular level, two types of renal-hepatic-pancreatic dysplasias have been described: (MIM 208540) a homozygous or compound heterozygous mutation in the NPNP3 gene on chromosome 3q22 and (MIM615415), a homozygous mutation in the NEK8 gene on chromosome 17q11 [31]. This group studied three fetuses from a consanguineous couple. They performed a whole-genome search for linkage by homozygosity mapping using the 250 Affymetrix single nucleotide polymorphism (SNP) array. The fetuses had multiple anomalies including cystic, dysplastic kidneys, and congenital hepatic fibrosis. One fetus had asplenia and situs inversus, and two had congenital heart disease.

The kidneys in RHPD and polysplenia tend to be grossly more solid than kidneys with multicystic dysplasia (Figure 9). We performed an autopsy on a child with polysplenia who also had renal dysplasia and congenital hepatic fibrosis (Figure 10).

\section{Hereditary Renal Adysplasia or Renal Hypodysplasia/aplasia (RHDA)}

This condition is autosomal dominant with variable penetrance. This entity was first described in 1973 by Buchta and collaborators [32] (included in the list of collaborators are Dr. Enid Gilbert and Dr. John Opitz). Pathologically, it is characterized by unilateral renal agenesis and contralateral dysplasia.

Renal agenesis and dysplasia were considered sporadic malformations by pediatric pathologists in the past; however, we have subsequently learned that they are hereditary and there are reports of parents with unilateral agenesis and siblings with either renal dysplasia or renal adysplasia. The combination of unilateral agenesis and dysplasia is perinatally lethal due to oligohydramnios sequence. So far, it is known that the disease can be produced by two types of mutations: RHDAl is caused by a homozygous or compound heterozygous mutation in the ITGA8 gene on chromosome 10p13, and RHDA2 is caused by a mutation in the FGF20 gene on chromosome 8q22.

A series of 41 patients with either bilateral renal agenesis or dysplasia and with combination of agenesis/dysplasia was studied at Brigham and Women's Hospital [33]. The prevalence of disease was calculated as 1 case in 6369 births. The parents and siblings underwent renal ultrasonography and it was determined that three parents and two siblings had unilateral renal agenesis, two parents had double ureter, one parent had a renal cyst, one sibling had hydronephrosis, one parent had unilateral multicystic kidney, two mothers had bicornuate uterus, and one had an absent ovary.

The authors concluded that parents of children with renal agenesis and/or dysplasia should know that they have an increased risk of having another severely affected child. The parents and siblings have an increased risk of having silent genitourinary 

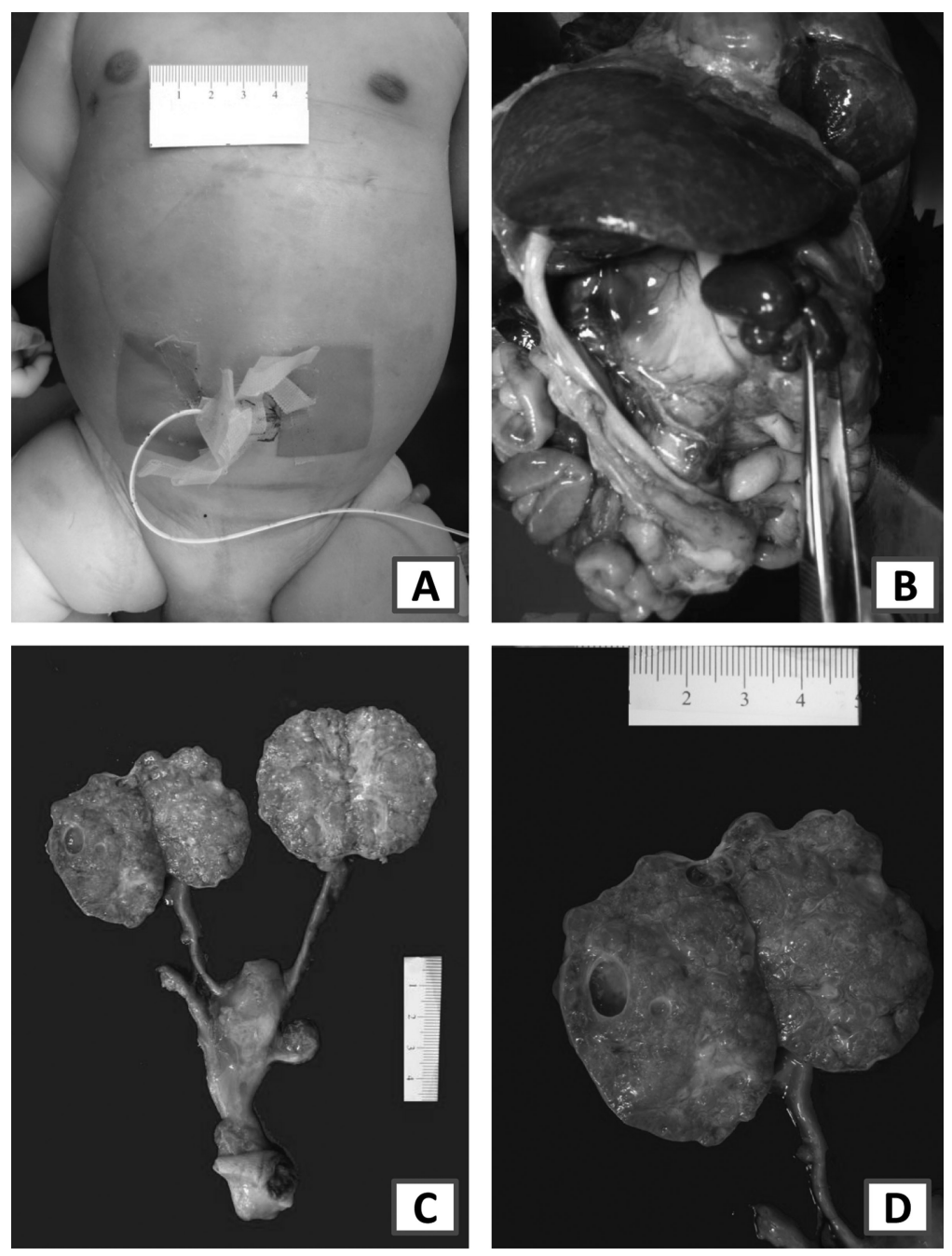

Figure 9. Nonobstructive renal dysplasia-polysplenia syndrome. (A) The only apparent body anomaly was a distended abdomen, but the child did not have a hypoplastic thorax. (B) There were several small splenicula at the left upper quadrant. The liver was moved up to allow better visualization and the forceps are holding the splenicula. (C) Both kidneys were dysplastic; however, the ureters were of normal caliber and the renal pelvis was not dilated. (D) Close-up view from the left kidney discloses lack of demarcation between cortex and medulla and several small cysts scattered throughout the parenchyma.

malformations; therefore, all first-degree relatives of affected children with renal agenesis or dysplasia should have an ultrasound to exclude a silent malformation.

\section{Zellweger Syndrome and Peroxisomal Disorders}

Peroxisomes are ultrastructural organelles that contain enzymes required to carry out critical cellular processes such as biosynthesis of membrane phospholipids, cholesterol, and bile acids. Peroxisomes also intervene in amino acid degradation to obtain glucose and energy, reduction of hydrogen peroxide by catalase, and prevention of excess synthesis of oxalate, which can result in formation of excessive calcium oxalate leading to nephrolithiasis. They also intervene in very long chain fatty acid degradation 

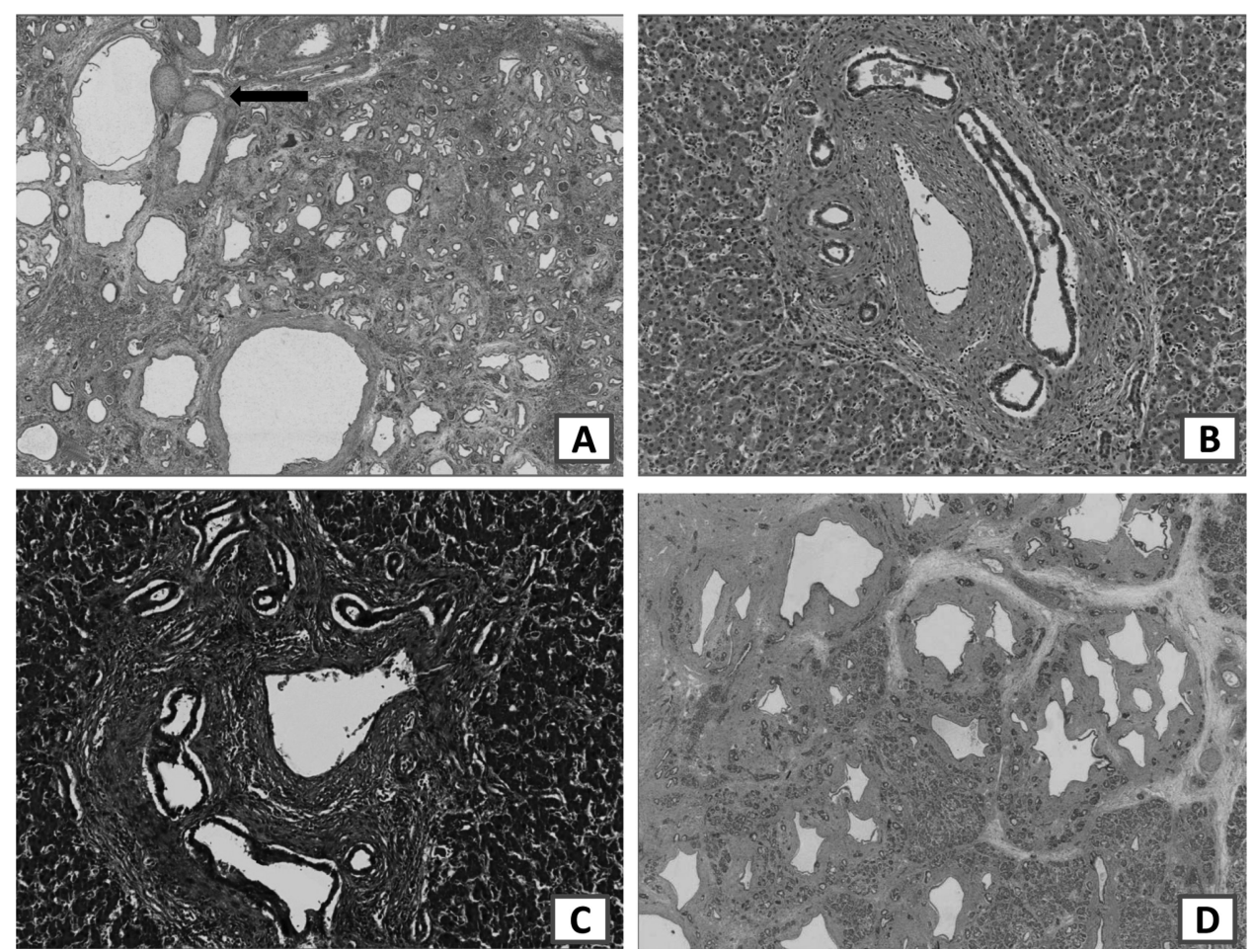

Figure 10. Photomicrographs from kidneys, liver and pancreas from the same patient shown in figure 9. (A) H\&E-stained kidney section with $10 \times$ lens, original magnification. Notice two islands of cartilage at the left upper corner (arrow). There is total architectural disorganization and multiple tubules are dilated. (B) H\&E-stained liver section with $10 \times$ lens, original magnification. The portal triad contains an expanded portal plate with numerous dilated bile ducts toward the periphery of the plate. The histology is diagnostic of a portal plate malformation. (C) Trichrome-stained liver section with $10 \times$ lens, original magnification. Notice the blue staining of the portal plate, the vein at the center and also numerous dilated, irregular bile ducts at the periphery. (D) Pancreatic cystic dysplasia (H\&E-stained section, $2.5 \times$ lens). The changes were focal with areas of normal histology and other parts containing cystic ducts. The photomicrograph was taken at the transition between normal and dysplastic pancreas.

and myelin formation; hence the neurological impairment. Peroxisomal disorders are divided into two major categories:

1. Peroxisome biogenesis disorders: Result in a failure to form intact, normal peroxisomes producing multiple metabolic abnormalities. They include Zellweger syndrome (ZS), also known as cerebrohepatorenal syndrome, and neonatal adrenoleukodystrophy (NALD) which is usually lethal within the first 10 years of life and is one of the few X-linked recessive peroxisomal disorders. Infantile Refsum disease that is milder than ZS and NALD, but fatal before age 20. Rhizomelic chondrodysplasia punctata is characterized by shortening of proximal extremities, broad nasal bridge, epicanthus, high-arched palate, dysplastic ears, and micrognathia.

2. Single-peroxisomal enzyme deficiencies: produce less severe clinical manifestations. Examples include acatalasia, pipecolic acidemia, hyperoxaluria type 1, acylCoA oxidase deficiency, etc. 
Zellweger is an uncommon autosomal recessive, lethal condition with average survival of less than 1 year. It has an incidence of 1 in 25000 or 50000 and it is characterized by hypotonia, long and narrow forehead, small whitish or grayish-brown spots in the iris (Brushfield spots), hypoplastic supraorbital ridges, epicanthal folds, abnormal brain gyri, and seizures. These children also have renal cystic dysplasia, persistent fetal lobulations, horseshoe kidney and urethral duplication. Ultrastructural examination of the liver and kidney in two patients with Zellweger demonstrated absent peroxisomes and abnormalities in mitochondria, the two organelles more related to energy metabolism. They also found either absent or extremely scarce smooth endoplasmic reticulum [34]. These authors described that by light microscopy, the kidneys contained small cortical cysts and occasionally dilated tubules.

\section{Trisomies and Congenital Anomalies of the Kidneys}

Renal anomalies are not a constant feature of trisomies; however, numerous examples have been reported in the literature of children with trisomies 13, 18, and 21 who also have hydronephrosis, horseshoe kidney, duplex kidney/collecting system, cortical cysts and/or cystic dysplasia, glomerular microcysts, or renal hypoplasia [35]. Egli and Stalder reviewed the available series, added their own cases, and concluded that there is an increased incidence of gross malformations of the urinary system in trisomies 13 and 18 . They found that in trisomy 21, the incidence is lower and stated that no specific renal or urinary tract malformation can be correlated to a specific chromosome or chromosome region [36].

Trisomy 13: (Patau syndrome) is a lethal chromosomal abnormality characterized by holoprosencephaly, arhinencephaly, microphthalmus, cleft lip and/or palate, polydactyly, and congenital heart disease. However, associated renal anomalies can be found at autopsy. We examined a fetus that was aborted because the amniocentesis demonstrated an extra chromosome 13. In addition to holoprosencephaly, he had absent crista galli, post-axial polydactyly, and cystic dilatation of renal tubules (Figure 11). The most common renal anomaly found in the review of 59 patients by Egli and Stalder was multicystic renal cortex (34\%) followed by hydronephrosis (21\%). There are case reports describing the common and uncommon features of trisomy 13 . These children are frequently born preterm and may have scalp defects, congenital heart disease, and Meckel's diverticulum. A patient with trisomy 13 and bilateral renal dysplasia has been described [37]. The patient had ectopic splenic tissue in the pancreas and a complex congenital heart disease.

Trisomy 18: Primarily affects the cardiovascular system and is better known by its association to ventricular (73\%) and atrial septal defects (41\%), patent ductus arteriosus, and pulmonary valve anomalies. Its characteristic facial features are a prominent occiput, narrow bifrontal diameter, low-set, malformed ears, small oral opening, overlapping of the fingers, and narrow pelvis. On the other hand, trisomy 18 tends to have almost the same incidence of renal anomalies as trisomy 13. The most common malformations are duplication of the collecting system and horseshoe kidney (25\%). Less commonly identified are cortical cysts (17\%) and hydronephrosis (15\%) [38]. One of our patients with trisomy 18 underwent autopsy at 7 days and besides the characteristic facial features, she had agenesis of corpus callosum, overlapping of the fingers and multicystic dysplastic kidneys (Figure 12).

Trisomy 21: May have renal involvement ranging from cortical microcysts, simple cysts, renal hypoplasia, and immature glomeruli deep in the cortex. These children may also develop obstructive uropathy. The largest autopsy series reported 124 fetuses and children with Down syndrome from three medical centers that underwent autopsies [39]. These authors found renal hypoplasia in 18 cases, glomerular microcysts in $24 \%$ of children, only one of these patients had an anatomical obstruction of the 

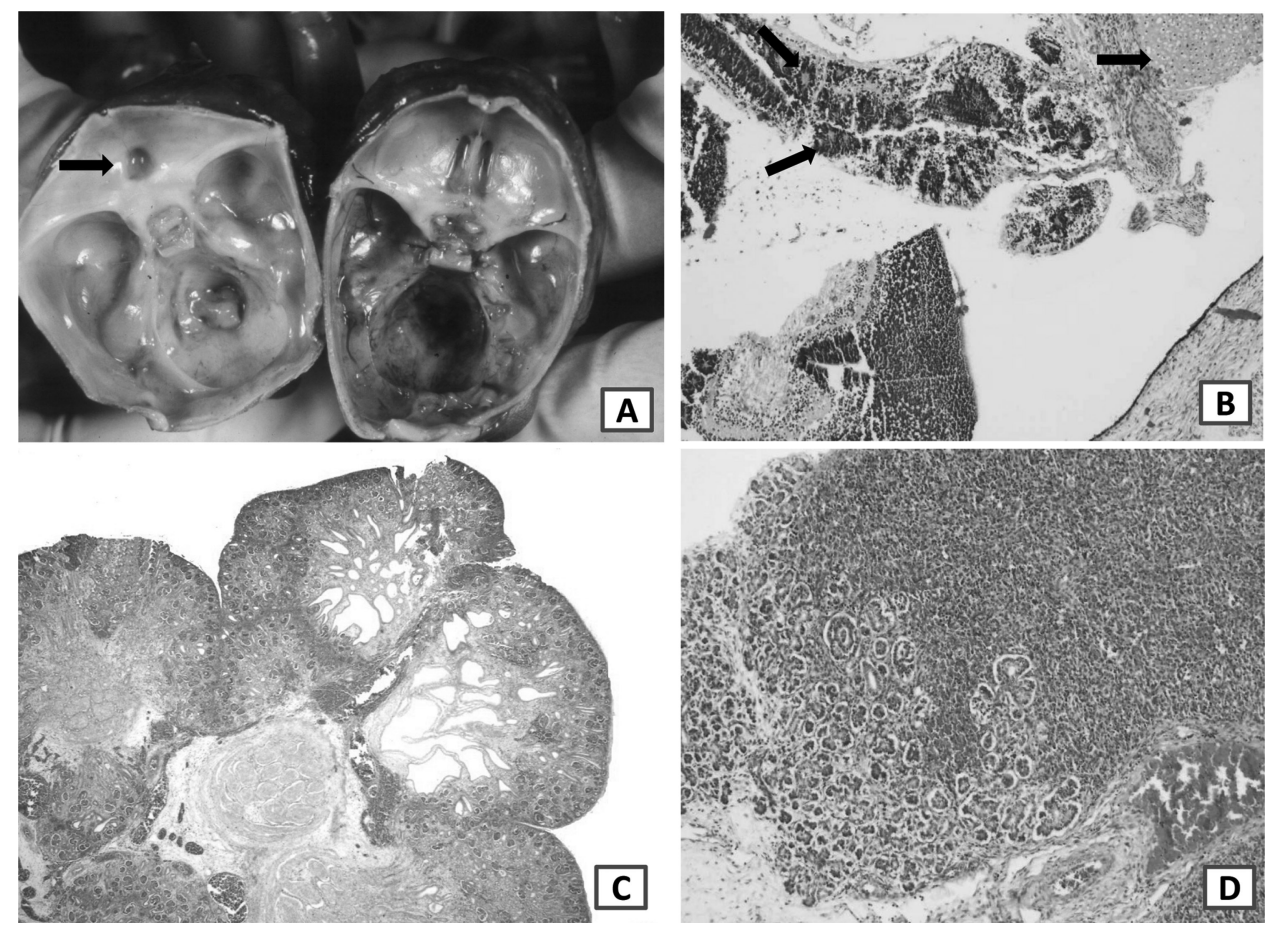

Figure 11. Fetus with trisomy 13, holoprosencephaly, and renal dysplasia. (A) Cranial bases from two fetuses: to the left the fetus with trisomy 13, holoprosencephaly, and arhinencephaly; to the right a fetus aborted because of trisomy 21. To the left, there is absence of crista galli (arrow). Compare with the fetus to the right that has a normal crista galli. (B) H\&E-stained section from the fetal retina $(10 \times$ lens $)$ demonstrates the presence of cartilage and multiple neuronal rosettes diagnostic of retinal dysplasia (arrows). (C) H\&E-stained renal section with $2 \times$ lens, original magnification. There are numerous dilated tubules at the corticomedullary junction and three nodules of dysplastic renal parenchyma in the medulla. (D) A small nodule was noticed in the spleen that was sampled and histologically was a splenopancreatic fusion (H\&E-stained section from the spleen,10× lens).

urinary tract, focal tubular dilatation in 10 cases, and 7 cases with simple cysts. Immature glomeruli deep into the cortex were present in 18 children (15\%). There were eight patients with obstructive uropathy (6\%). Four of them had cystic renal dysplasia and the other four had a combination of hydronephrosis and hydroureter.

\section{VACTER-L Association}

This acronym stands for vertebral anomalies, anal, and cardiac malformations, tracheo-esophageal (TE) fistula/atresia, radial and renal anomalies, and limb malformations. The majority of experts in VACTER-L require at least three of the abovementioned anomalies to establish the diagnosis. When there is esophageal atresia, the first intrauterine manifestation is polyhydramnios. TE fistula can be documented at autopsy and renal dysplasia is common in these children (Figure 13). The urologic implications of the VATER syndrome were reported by Uehling and collaborators (Dr. Enid Gilbert included) [39]. They studied 23 children with the VATER association and found that 21 had significant genitourinary involvement.

The differential diagnosis includes Baller-Gerold syndrome (certain facial features, and radial aplasia or hypoplasia), CHARGE syndrome (besides choanal atresia and coloboma can have cardiac and genitourinary anomalies), Currarino syndrome (lumbosacral malformations, constipation and renal abnormalities), deletion 22q11.2 

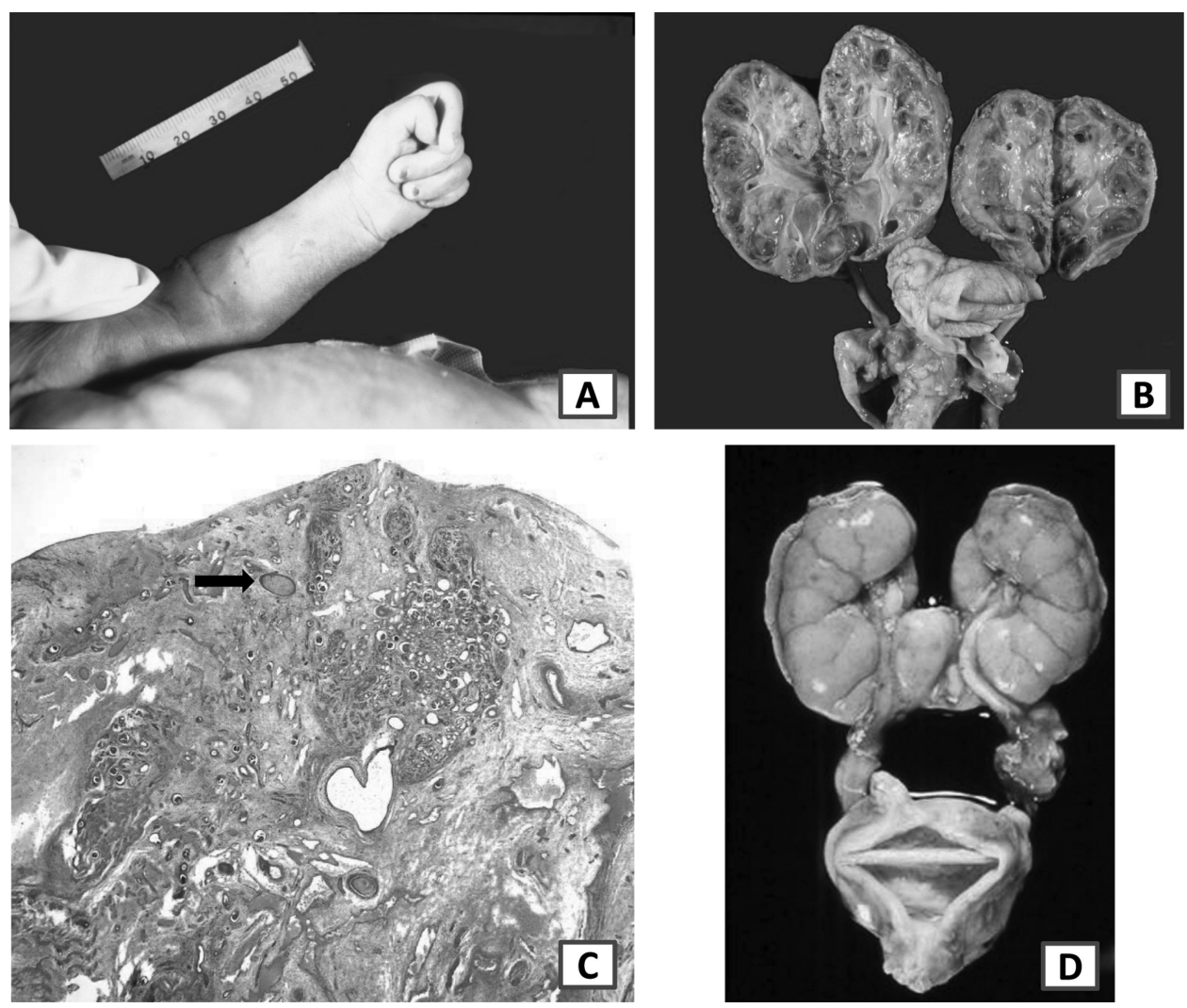

Figure 12. Renal anomalies in trisomy 18. (A) Neonate with trisomy 18 and overlapping of the fingers associated with fingernail hypoplasia. (B) Kidneys from the same patient with nonobstructive renal dysplasia. (C) H\&E-stained renal section with $2 \times$ lens, original magnification. Observe a small island of cartilage and disorganized renal parenchyma. (D) Another patient with trisomy 18 and horseshoe kidney. The neonate had narrow hips that explain the fusion at the lower poles.

syndrome (cardiac, vertebral and renal anomalies), Fanconi anemia (radial hypoplasia/aplasia, thumb anomalies and hematological conditions), Feingold syndrome (hypoplastic thumb, renal and cardiac congenital disease), Fryns syndrome (congenital diaphragmatic hernia, cardiac disease and hypoplastic thumb), MURCS association (Mullerian duct, renal agenesis, and cervical vertebral defects), oculoauriculovertebral syndrome (vertebral anomalies hypoplasia of maxilla and mandible and ear anomalies), Pallister-Hall syndrome (abnormal digits and imperforate anus), Townes-Brocks syndrome (thumbs, auricular and anal anomalies), and VACTERL with hydrocephalus [40]. A careful physical examination and family history are helpful to narrow down which conditions are most likely in a patient with features suggestive of VACTERL association. For example, autosomal dominant inheritance of certain features may suggest Townes-Brocks syndrome, and the presence of other features not typically seen in VACTER-L association may hint towards other disorders, such as pigmentary abnormalities in Fanconi anemia, or hypocalcemia in deletion 22q11.2 syndrome. A panel of experts published an excellent article about the approach to confirm suspected VACTER [41]. According to them the initial work up should include a complete family history and physical examination, a supine anteroposterior (AP) chest radiograph, supine AP and lateral views of the entire vertebral column, including sacral views, and a transthoracic echocardiogram to look for congenital heart disease. In 

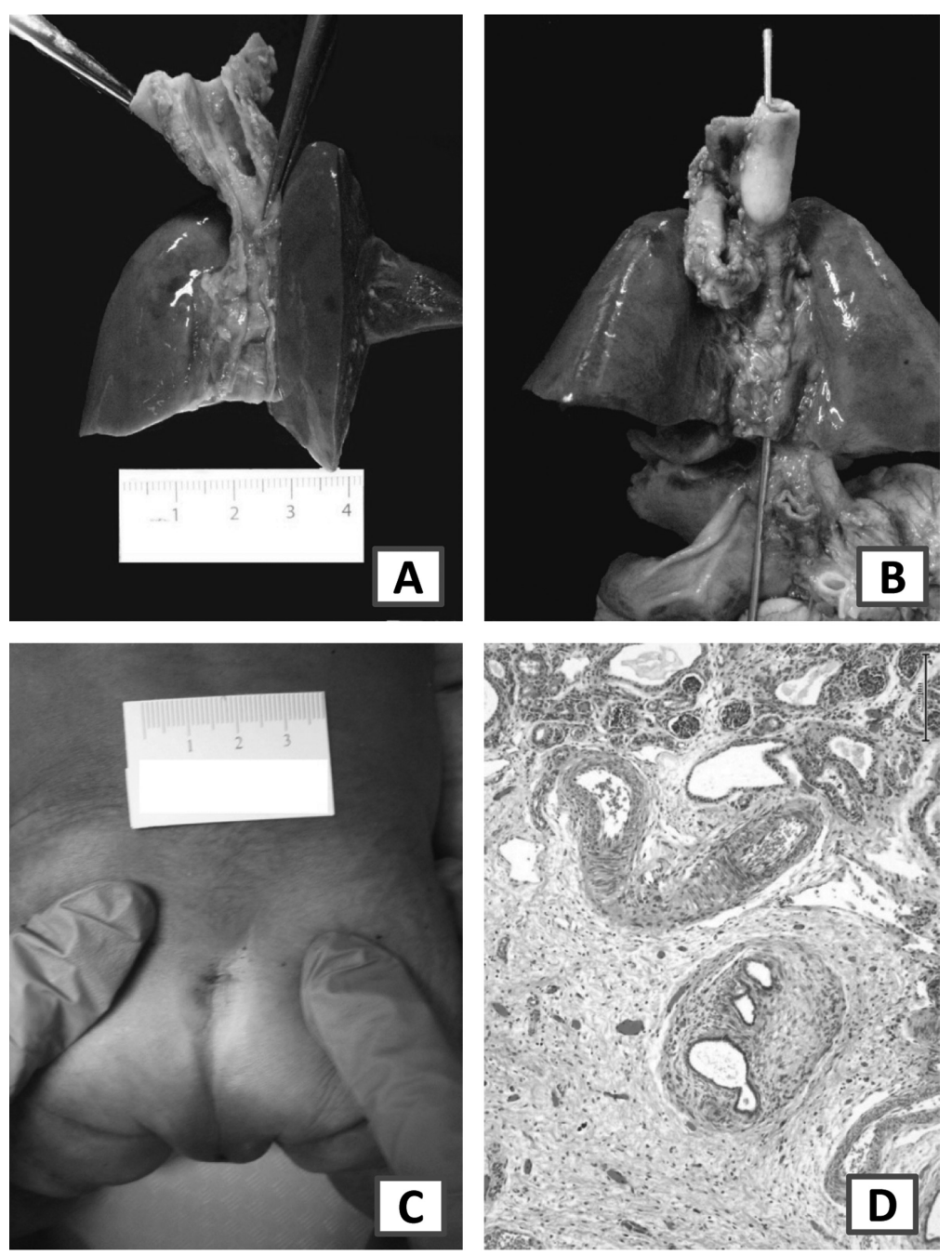

Figure 13. VACTER association. (A) The trachea was open posteriorly to demonstrate a tracheoesophageal fistula. (B) The photograph depicts esophageal atresia and tracheo-esophageal fistula. The probe was entered in the trachea and came out at the gastroesophageal junction. (C) Neonate with imperforate anus. (D) H\&E-stained renal section with $10 \times$ lens, original magnification. There are several normal glomeruli at the upper part of the photograph, but they are intermixed with cystic tubules and in the lower half there are three cysts surrounded by concentric fibrosis.

patients with increased oral secretions, chocking with feeds, or respiratory distress, an attempt to pass a nasogastric tube will help to confirm or exclude esophageal atresia. Anorectal malformations can be detected by physical examination in the majority of cases, but to better determine the extent of anomalies, an abdominal ultrasound should be performed. Radial and thumb anomalies are usually detected by simple inspection, but radiologic examination of the affected extremity will better delineate the malformation. In order to exclude Fanconi anemia, a complete blood cell count is extremely helpful.

\section{Bilateral Renal Agenesis (Potter Syndrome)}

Complete absence of both kidneys is one of the most severe congenital renal anomalies that had been recognized since the 18th century. However, it wasn't until 1946 
when Dr. Edith Potter published the article entitled "Bilateral Renal Agenesis" [42] where she defined the reduced amount or absence of amniotic fluid, and stated "If more definite information concerning the amniotic fluid had been available it might have been possible to shed some light on the importance of the secretion by the fetal kidneys in maintaining a normal volume of amniotic fluid." Dr. Potter also noticed "two anomalies were observed in almost all the infants which to my knowledge have not previously been recognized as characteristically associated with renal agenesis. These are hypoplasia of the lungs and an abnormal facial expression."

It is now widely recognized that the so-called "Potter's face" with folds under the eyes, flat nose, receding chin, and low-set ears as well as pulmonary hypoplasia, wide hands, and rocker-bottom feet are mechanical deformities produced by the oligohydramnios sequence (Figure 14).

This year a group from France, with collaboration from Australia, published an elegant study using a whole-exome sequencing approach in families that had more than one fetus with bilateral renal agenesis [43]. There is an Itga8 knock-out mouse model with bilateral renal agenesis that was one of the favorite candidates. They identified recessive mutations of the integrin $\alpha 8$-(ITGA8) encoding gene in two families (one of them consanguineous). This gene has a crucial role in renal development and it is expressed in the metanephric mesenchyme surrounding the ureteric bud. The authors concluded that bilateral renal agenesis can be an autosomal recessive disorder.

\section{Caudal Dysplasia Syndrome}

This syndrome is characterized by hypoplastic lower extremities, caudal vertebrae, sacrum, neural tube, and urogenital system. They also have an imperforate anus. Renal anomalies include bilateral renal agenesis, renal dysplasia, and horseshoe kidney [44] (Figure 15). Frequently, their placentas have a single umbilical artery and less commonly have amnion nodosum secondary to oligohydramnios. These patients may also have congenital heart disease and their mothers frequently have pregestational diabetes mellitus. The association of polysplenia and caudal dysplasia has been reported [45].

Infants of diabetic mothers (IDM) have three to four times the incidence of congenital malformations than that in the general population. They can have skeletal, neurologic, genito-urinary and digestive tract maldevelopment, and caudal dysplasia syndrome. The brain and proximal spinal cord are normal in children with caudal dysplasia. Surviving children usually have a normal intelligence, but tend to have lower extremity difficulties as well as urinary problems and may require extensive intervention by pediatric orthopedics and urologists. Prenatal diagnosis can be made by fetal ultrasound and parental counseling should be offered depending on the severity of malformations.

As a general rule, infants of diabetic mothers (IDM) have three to four times the incidence of congenital malformations than that in the general population. IDM can have skeletal, neurologic, genitourinary as well as digestive tract maldevelopment, and caudal dysplasia syndrome. The brain and proximal spinal cord are normal in children with caudal dysplasia. Surviving patients usually have a normal intelligence, but tend to have lower extremity difficulties as well as urinary problems and may require extensive intervention by specialties such as pediatric orthopedics and urologists. Prenatal diagnosis can be made by fetal ultrasound and parental counseling should be offered depending on the severity of malformations as many of these neonates do not survive the perinatal period due to pulmonary hypoplasia. A newborn boy infant of a diabetic mother with poor control was described by Dr. Enid Gilbert and collaborators in Wisconsin [46]. The neonate had hydrocephalus due to Dandy Walker 

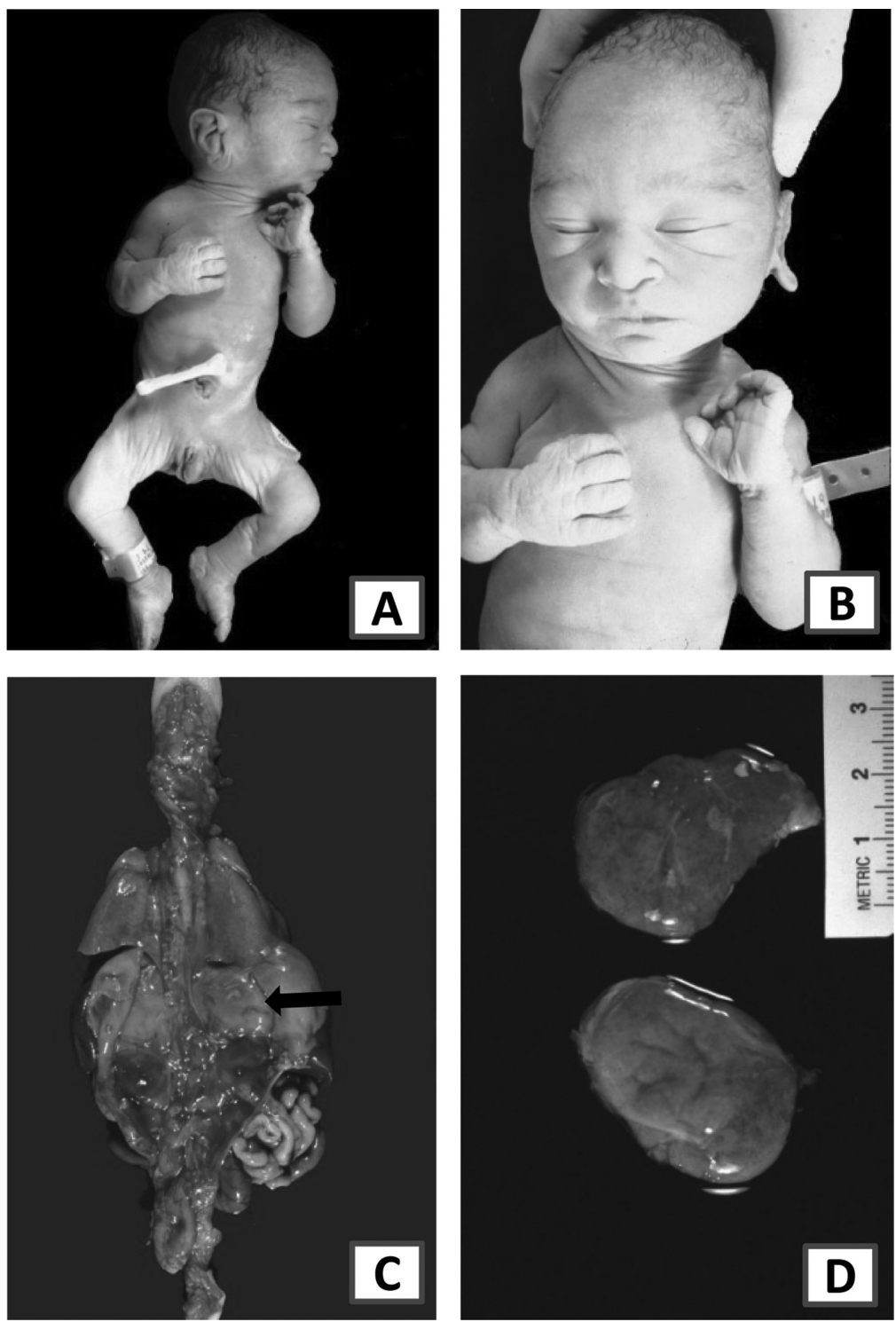

Figure 14. Bilateral renal agenesis (Potter sequence) (A) Newborn boy with characteristic Potter face with low-set, pliable ears, parrot-beaked nose, and receding chin. (B) Frontal view from the neonate's face and upper body discloses fold under the eyes, low-set ears, flat nose, and broad hands. (C) Posterior view from organ block shows hypoplastic lungs and flat adrenal glands (arrows). Both kidneys were absent. (D) Dissected flat adrenal glands characteristic of renal agenesis.

malformation with a posterior fossa cyst, dilatation of the 3rd and 4th ventricles and cerebral aqueduct, hypertelorism, abnormal asymmetrical ears, a right with preauricular skin tag, absence of external auditory meatus, severe micrognathia, cleft lip and cleft palate, broad flat nose, bilateral upper limb amelia, asymmetric thorax, undescended testes, abnormal scrotum, short bowed lower limbs, and right calcaneovalgus with absence of all digits except for hallux. Radiologic examination demonstrated, among many anomalies, absence of lower sacral and coccygeal elements. However, his kidneys were normal. 

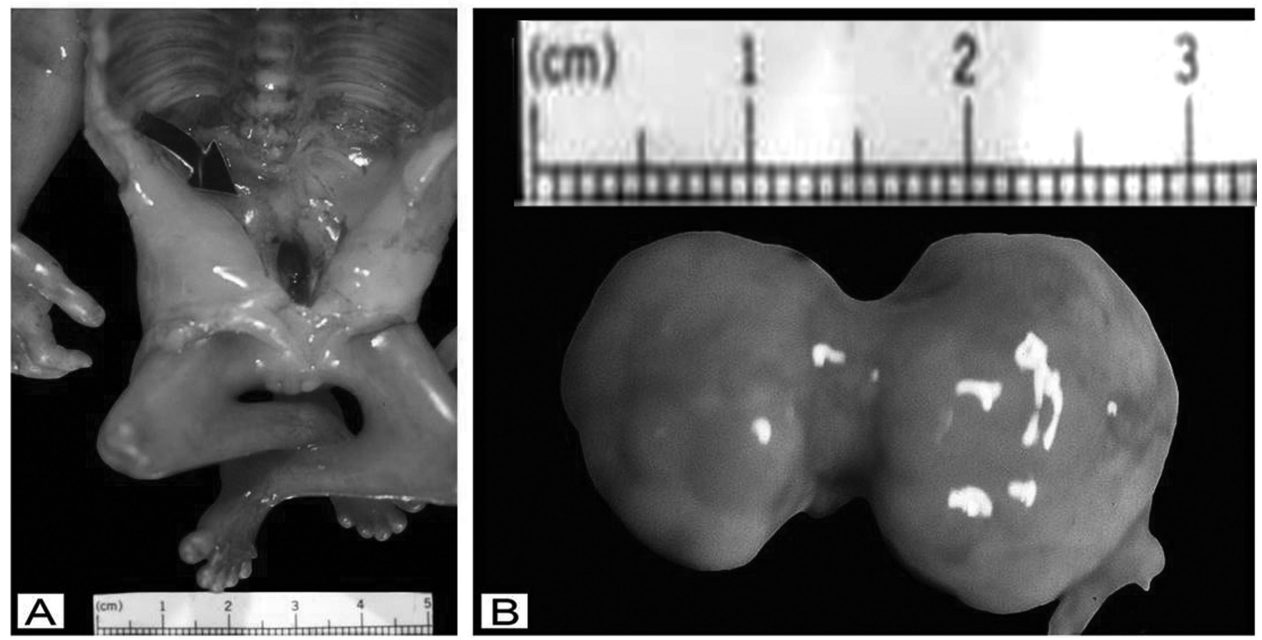

Figure 15. Caudal dysplasia syndrome (A) Open and eviscerated body of a fetus a 21 weeks. Black arrow points to absent sacrum. Both lower extremities are hypoplastic and contracted. (B) Horseshoe kidney from the same fetus.

\section{Sirenomelia}

This developmental defect involving the caudal region of the body is named after the Latin word siren (Greek sirein) which means mermaid and melia (in Greek limb). The common problem of these fetuses and children is they have a single lower extremity (Figure 16). The majority of them have imperforate anus, either ambiguous or absent external genitalia, and single umbilical artery. The spectrum of renal anomalies includes bilateral or unilateral renal agenesis with absent ureters and urinary bladder, renal dysplasia and renal hypoplasia. They have the oligohydramnios sequence with flat nose, receding chin, low-set ears, hypoplastic thorax, and broad hands. The majority of neonates affected by sirenomelia die in the neonatal period of respiratory insufficiency. There is still controversy in the literature about the theory that caudal dysplasia or dysgenesis and sirenomelia constitute part of the same disease with several degrees of expression or if they are two separate entities with completely different etiologies. A group from Australia [47] postulates that the considerable overlap between ultrasonographic and pathological features of both caudal dysgenesis and sirenomelia support the theory of early deficient mesodermal development. They also believe that the association between caudal dysgenesis and VACTERL probably explains a common pathogenetic mechanism involving developmental anomalies of mesoderm. However, they also noticed that maternal diabetes is more commonly associated with caudal dysplasia than sirenomelia.

Radiologic examination discloses several possibilities including rudimentary femur and proximal tibia, or more normal long bones, eversion, retroversion, and fusion of the feet, or unusually separate feet. They may have vertebral and pelvic anomalies including hypoplastic sacrum, hypoplastic iliac wings, absent pubic bones, etc.

\section{Autosomal Dominant Polycystic Kidneys (ADPK)}

This relatively common condition affects 1 of every 400 to 1000 live births and accounts for approximately $10 \%$ of patients with chronic renal failure requiring dialysis or transplant. ADPK should be considered a systemic disorder that mainly affects adult patients. They also develop hepatic and pancreatic cysts, chronic hypertension, intracranial aneurysms, and cardiac valve anomalies especially mitral valve prolapse. The 

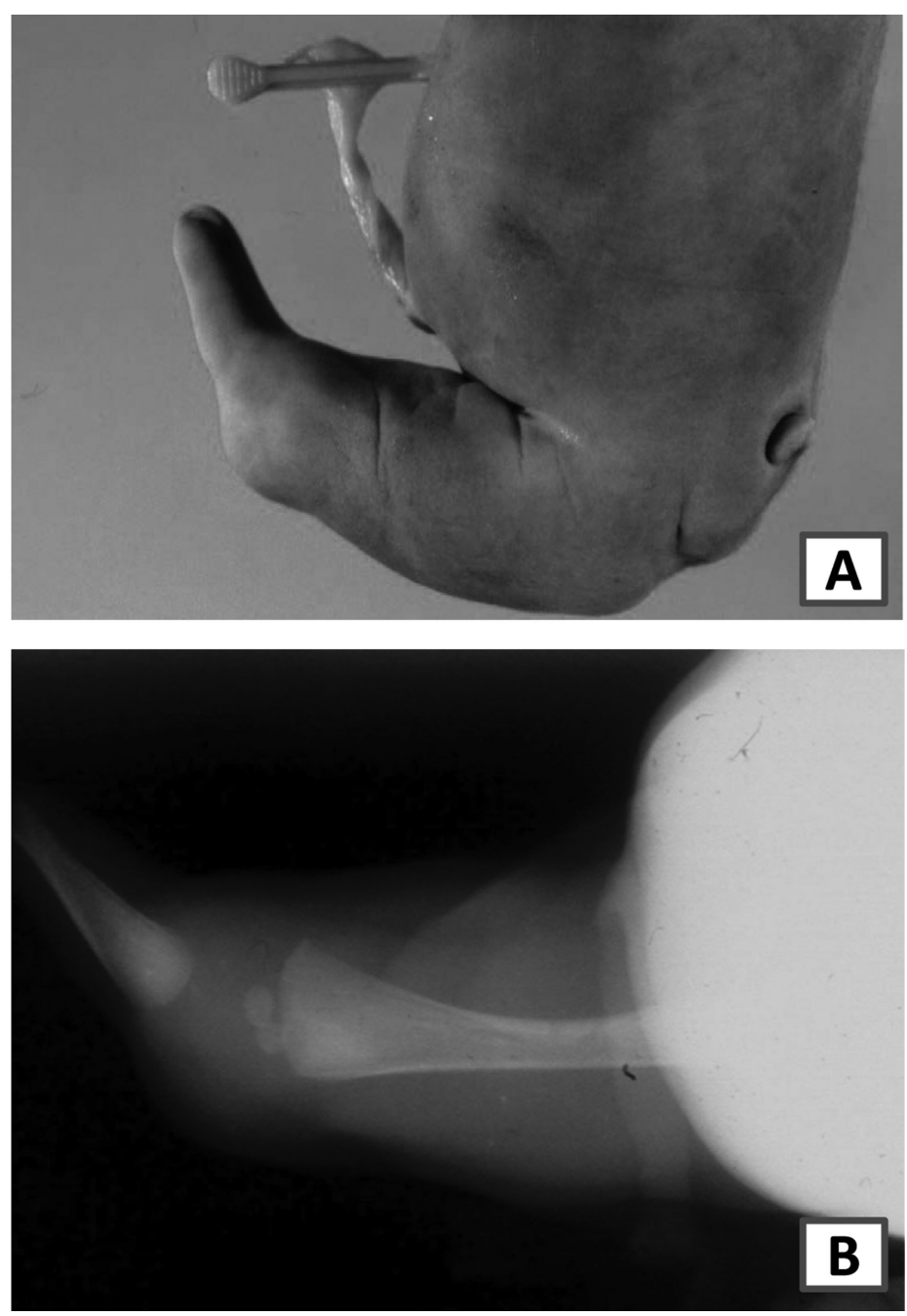

Figure 16. Sirenomelia (A) Lateral view of lower body. This fetus had absent external genitalia, imperforate anus with an anal tag, and a single lower extremity. (B) Lateral radiograph demonstrates a single femur and fibula. The foot was absent.

likelihood of renal failure increases progressively with age after 40 years, rising to $25 \%$ by age $50,40 \%$ at 60 years and $75 \%$ at age 70 . The kidneys of adult patients contain multiple round cysts of variable size filled with urine and blood become extremely large extending up to 10 times their normal weight. Histology reveals completely disorganized renal parenchyma with large cystically dilated tubules and occasional glomeruli and fibrotic interstitium with chronic inflammation (Figure 17). The disease is now considered one of the primary ciliopathies.

Every renal cell in patients with ADPK carries a copy of the mutated PKD1 gene; however, cysts appear to develop in relatively few tubules, mainly the collecting ducts. Polycystin-1 is a protein that has a role in establishing and maintaining a normal tubular phenotype and in injury repair. The allele from the normal parent usually produces enough polycystin-1 to maintain the tubules relatively healthy for many years. However, later in life some cells undergo somatic mutations that render the normal allele 

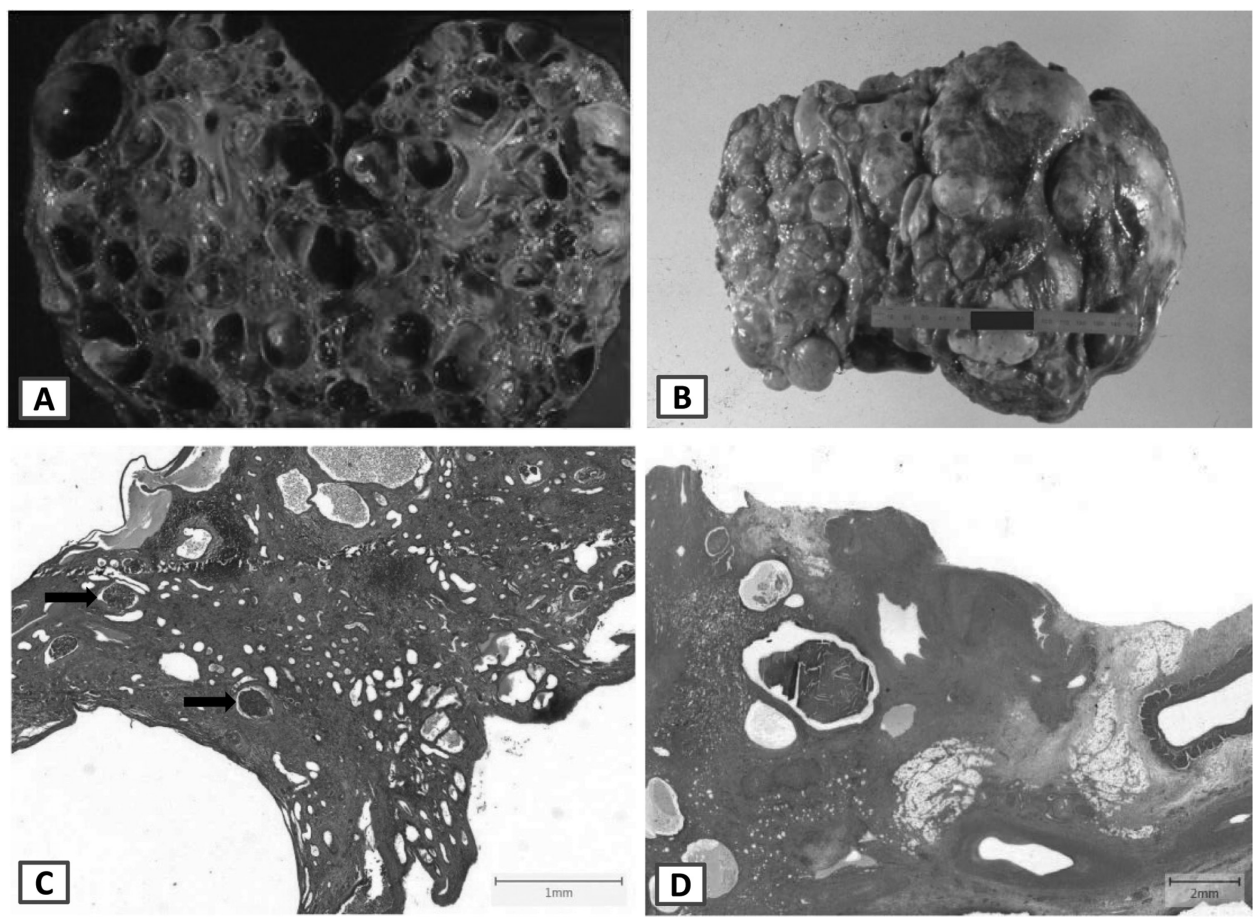

Figure 17. Autosomal dominant polycystic kidneys and liver in an adult. (A) The kidneys were massively enlarged weighing 10 times the expected weight. The cut section reveals numerous round cysts in cortex and medulla. (B) The liver also contains multiple cysts involving almost the entire parenchyma. (C) Histologic section from the kidney (H\&E stained) shows a few scattered glomeruli (arrows) and two large cysts at the bottom of the photomicrograph. One of the cysts is surrounded by chronic inflammation. Internal scale $1 \mathrm{~mm}$. (D) This section depicts less glomeruli and more interstitial fibrosis. Internal scale $2 \mathrm{~mm}$.

incompetent, and polycystin-1 levels fall below the required amount to maintain the balance [48].

For many years, ADPK was considered an untreatable renal disease leading to chronic renal failure and required either dialysis or transplant, but more recently there is some hope of a better treatment by early administration of an AVP (argininevasopressin) $-\mathrm{V}_{2}$ receptor inhibitor in individuals with subclinically detected disease [49]. The rationale behind this therapy is that the collecting ducts and distal nephrons (that are the more severely affected by cysts in ADPK) are sensitive to vasopressin. This receptor is the main hormonal regulator of adenyl cyclase activity in collecting ducts. To avoid dehydration mammals live under the constant action of AVP on the distal nephron and collecting duct. When the individual drinks large volumes of water, plasma AVP levels decrease enough to make the urine more dilute than plasma; therefore, during most of the day, cyst epithelial cells undergo stimulation to secrete fluid. The circulating levels of AVP are likely elevated in patients with ADPK to compensate for the reduced concentrating capacity of the affected kidneys and the AVP effect leads to cyst formation. The administration of an arginine-vasopressin receptor inhibitor delays the cyst formation, but does not help regenerating tubular epithelium; therefore it is critical to start treatment as early as possible in order to prevent cyst formation. Other recommendations are to drink plenty of fluids up to $3 \mathrm{~L} /$ day for adults and proportionately less for children, avoid caffeine, decrease salt and protein intake, add ACE (angiotensin-converting enzyme) inhibitors or ARBs (angiotensin-receptor 
blocker) to lower the blood pressure, to decrease the amount of fat ingested, and prescribe a cholesterol lowering agent.

\section{Autosomal Recessive Polycystic Kidneys (ARPK)}

This more uncommon disease is currently considered a primary ciliopathy that equally affects the kidneys and liver. ARPK has an incidence of 1:20 000 to 1:40 000 live births and a heterozygous carrier rate of 1 in 70 . It occurs as a result of a mutation in a single gene named Polycystic Kidney and Hepatic Disease (PKHD1) [50]. Severely affected fetuses are born with oligohydramnios, Potter face, and some will develop respiratory insufficiency, but many survive the neonatal period. Of all neonatal survivors, approximately $40 \%$ have severe hepatic and renal disease. Of the remaining children, $30 \%$ present with severe renal and mild hepatobiliary disease and the other $30 \%$ with severe hepatobiliary problems and mild renal disease.

The renal pathology is extremely characteristic because it is always bilateral, the organ is enlarged but maintains its reniform shape, and when opened it transudates a lot of urine. Histologically, the cysts are elongated and their long axis is perpendicular to the capsule (Figure 18). The hepatic manifestations are congenital hepatic fibrosis (CHF), that is undistinguishable from the CHF found in other ciliopathies such as Meckel, short rib, or polysplenia. Secondary to the liver fibrosis they develop
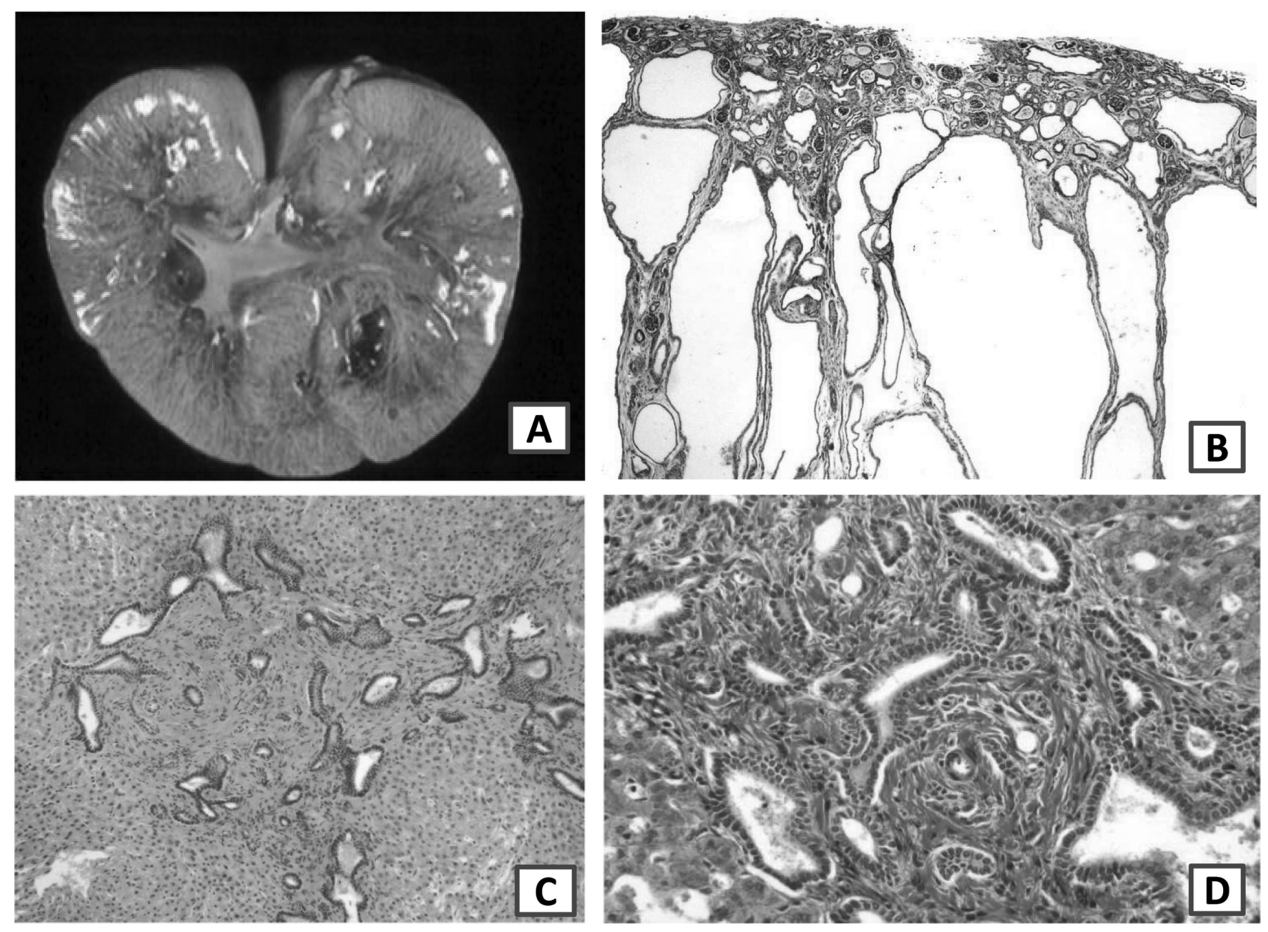

Figure 18. Autosomal recessive polycystic kidneys. (A) The kidneys maintain the reniform shape, but are extremely enlarged. The cut section discloses numerous elongated and urine-filled cysts with radial orientation. (B) H\&E-stained renal section with $2 \times$ lens, original magnification. There are preserved glomeruli, but the tubules are oriented with the longer axis perpendicular to the capsule. (C) The hepatic photomicrograph exhibits congenital hepatic fibrosis with numerous bile ducts with an open lumen in the portal plate $(4 \times$ lens, original magnification). (D) Trichrome stained section $(10 \times$ lens $)$ stains the portal plate in blue and bile duct epithelium in red. Surrounding hepatocytes at the upper right and lower left are normal. 
portal hypertension, esophageal varices, hemorrhoids, upper gastrointestinal bleeding, splenomegaly, and hypersplenism.

Genetic studies have demonstrated that ARPK is associated with two genes (PKD1 and PKD2). The former produces the severe form of the disease and its gene product, polycystin-1 (PC1), is a receptor-like integral membrane protein that seems to be involved in cell-cell matrix interactions and also plays a role in calcium homeostasis through its physical interaction with polycystin-2, the protein product of PKD2.

Parents of a child born with ARPK who are obligate carriers may be offered preimplantation genetic diagnosis using single-cell multiple displacement amplification (MDA) products for PKHD1 haplotyping, which significantly decreases the problem of allelic dropout. This specific protocol employs whole-genome amplification of single blastomeres, MDA, and haplotype analysis with 20 novel polymorphic short-tandem repeat (STR) markers from the PKHD1 gene and flanking sequences.

Once the child is born with the disease, the current treatment is symptomatic. However, in cases of severe renal and hepatobiliary disease, the combined renal-liver transplant looks promising because the outcome of liver transplant has improved recently and it has been proven that if left unattended, CHF may lead to ascending cholangitis, sepsis, portal hypertension and gastrointestinal bleeding. All of these complications could impact the ultimate outcome of liver transplant months or years after renal transplant. The future seems more promising for these patients as there are several pre-clinical trials that hopefully will discover new treatment modalities to block fluid transfer into the tubular epithelial cells and decrease or completely block cyst formation. The group of Drs. Sweeney and Avner [50] is currently using a compound that inhibits both pathways: the epidermal growth factor receptor (EGFR)-axis and the cyclic AMP or G-protein pathway via inhibition of c-Src in an attempt to be able to use a smaller dose to achieve maximum cyst reduction and to minimize drug toxicity.

\section{SUMMARY}

Congenital anomalies of the kidney and urinary tract continue to be a group of diseases with different degrees of severity and many of them require a multidisciplinary approach for accurate diagnosis and better treatment. Since many of these congenital anomalies are hereditary, advances in prenatal diagnosis, fetal surgery, combined liver and kidney transplants, as well as targeted therapies have improved the prognosis and quality of life in affected families.

For pediatric pathologists, it is essential to establish the correct diagnosis in order to facilitate adequate genetic counseling and to alert the families of other possible affected members that could benefit from earlier intervention.

\section{ACKNOWLEDGMENTS}

I thank Dr. Elizabeth Whittington from the Department of Pathology at the University of Miami, Miller School of Medicine for reviewing the manuscript and for her pertinent editorial comments.

\section{Declaration of Interest}

The authors report no conflicts of interest. The authors alone are responsible for the content and writing of the paper. 


\section{REFERENCES}

[1] Decter R. Renal duplication and fusion anomalies. Pediatr Clin North Am 1997;44:1323-1341.

[2] Atwell JD, Cook PL, Howell CJ, et al. Familial incidence of bifid and double ureters. Arch Dis Child 1974;49:390-393.

[3] Lacson A, Bernstein J, Risdon RA, Gilbert-Barness E. Renal system. Chapter 27. In: Potter's Pathology of the Fetus, Infant and Child. Gilbert-Barness E., ed. 2nd ed., Philadelphia, PA: Mosby, Elsevier, 2007.

[4] Hisano M, Denes FT, Brito AH, et al. Laparoscopic ureteropyeloanastomosis in the treatment of duplex system. Int Braz J Urol 2012;38:235-241.

[5] Williams B, Tareen B, Resnick MI. Pathophysiology and treatment of ureteropelvic junction obstruction. Curr Urol Rep 2007;8:111-117.

[6] Ruano-Gil D, Tejedo-Mateu A. Human embryo (12 mm) with mesohydronephrosis and ureterohydronephrosis. Acta Anat 1975;93:135-140.

[7] Aoki Y, Mori S, Kitajima, et al. Id2 haploinsufficiency in mice leads to congenital hydronephrosis resembling that in humans. Genes Cells 2004;9:1287-1296.

[8] Kajbafzadeh AM, Payabvash S, Salmasi AH, et al. Smooth muscle cell apoptosis and defective neural development in congenital ureteropelvic junction obstruction. J Urol 2006;176:718-723.

[9] Blanc T, Koulouris E, Botto N, et al. Laparoscopic pyeloplasty in children with horseshoe kidney. $J$ Urol 2014;191:1097-103.

[10] Tonni G, Ida V, Alessandro V, Bonasoni MP. Prune-belly syndrome: case series and review of the literatura regarding early prenatal diagnosis, epidemiology, genetic factors, treatment, and prognosis. Fetal Pediatr Pathol 2013;31:13-24.

[11] Granberg CF, Harrison SM, Dajusta D, et al. Genetic basis of prune belly syndrome: Screening for HNF1 $\beta$ gene. J Urol 2012;187:272-278.

[12] Oram RA, Edhill EL, Blackman J, et al. Mutations in the hepatocytes nuclear factor- $1 \beta$ (HNF1 $\beta$ ) gene are common with combined uterine and renal malformations but are not found with isolated uterine malformations. Am J Obstetr Gynecol 2010;203:364.e1-e5.

[13] Quintero RA, Gomez Castro LA, Bermudez C, et al. In utero management of fetal lower urinary tract obstruction with a novel shunt: a landmark development in fetal therapy. J Matern Fetal Neonatal Med 2010;23:806-12.

[14] Rojas CP, Urbiztondo AK, Bruce JH, Rodriguez MM. Comparative immunohistochemical study of multicystic dysplastic kidneys with and without obstruction. Fetal Pediatr Pathol 2011;30:209-19.

[15] Matsell DG, Bennett T, Armstrong RA, et al. Insulin-like growth factor (IGF) binding protein gene expression in multicystic renal dysplasia. J AM Soc Nephrol 1997;8:85-94.

[16] Yosypiv IV. Congenital anomalies of the kidney and urinary tract: a genetic disorder?. Int J Nephrol 2012;2012:909083. Epub 2012 May 20.

[17] Saisawat P, Tasic V, Vega-Warner V, et al. Identification of two novel CAKUT-causing genes by massively parallel exon resenquencing of candidate genes in patients with unilateral renal agenesis. Kidney International 2012;81:196-200.

[18] Tobin JL, Beales PL. The nonmotile ciliopathies. Genet Med 2009;11:386-402.

[19] Opitz JM, Schultka R, Göbbel L. Annals of morphology. Meckel on developmental pathology. Am J Med Genet 2006;140A:115-28.

[20] Salonen R, Paavola P. Meckel syndrome. J Med Genet 1998;35:497-501.

[21] Joubert M, Jean-Jacques E, Robb JP, Anderman F. Familial agenesis of the cerebellar vermis. A syndrome of episodic apnea, abnormal eye movements, ataxia, and retardation. Neurology 1969;19:813-825.

[22] Parisi MA. Clinical and molecular features of Joubert syndrome and related disorders. Am J Med Genet C Semin Med Genet 2009;151C:326-340.

[23] Cidecyan D, Rodriguez MM, Haun RL, et al. New findings in short rib syndrome. Am J Med Genet 1993;46:255-259.

[24] Thiel C, Kessler, Giessl A, et al. NEK1 mutations cause short-rib polydactyly syndrome type Majewski. Am J Hum Genet 2011;88:106-114.

[25] Davis EE, Zhang Q, Liu Q, et al. TTC21B contributes both causal and modifying alleles across the ciliopathy spectrum. Nat Genet 2011;43:189-196.

[26] Green JS, Parfrey PS, Harnett JD, et al. The cardinal manifestations of Bardet-Biedl syndrome, a form of Laurence-Moon-Biedl syndrome. N Eng J Med 1989;321:1002-1009.

[27] Gershoni-Baruch R, Nachlieli T, Leibo R, et al. Cystic kidney dysplasia and polydactyly in 3 sibs with Bardet-Biedl syndrome. Am J Med Genet 1992;44:269-273.

[28] Ivemark BL, Oldfelt V, Zetterstrom R. Familial dysplasia of kidneys, liver and pancreas: a probably genetically determined syndrome. Acta Paediatr 1959;48:1-11.

[29] Crawfurd MD. Renal dysplasia and asplenia in two sibs. Clin Genet 1978;14:338-344. 
[30] Bernstein J, Chandra M, Creswell J, et al. Renal-hepatic-pancreatic dysplasia: a syndrome reconsidered. Am J Med Genet 1987;26:391-403.

[31] Frank V, Habbig S, Bartram MP, et al. Mutations in NEK8 link multiple organ dysplasia with altered Hippo signaling and increased c-MYC expression. Hum Molec Genet 2013;22:2177-2185.

[32] Buchta RM, Viseskul C, Gilbert EF, et al. Familiar bilateral renal agenesis and hereditary renal adysplasia. Z Kinderheilkd 1973;115:111-129.

[33] Roodhoof AM, Benholtz JC, Holmes LB. Familial nature of congenital absence and severe dysgenesis of both kidneys. N Eng J Med 1984;310:1341-1345.

[34] Goldfisher S, Moore CL, Johnson AB et al. Peroxisomal and mitochondrial defects in the cerebrohepato-renal syndrome. Science 1973;182:62-64.

[35] Amor DJ, de Crespigny L, McKinlay Gardner RJ. Urinary tract defects and chromosomal disorders. Chapter 5. In: The Genetics of Renal Disease. Flinter F, Maher E, Saggar-Malik A. ed. 1st ed., New York, NY: Oxford University Press; 2003. p. 117.

[36] Egli F, Stalder G. Malformations of kidney and urinary tract in common chromosomal aberrations. Humangenetik 1973;18:16-32.

[37] Balci S, Güçer S, Orhan D, Karagöz T. A well-documented trisomy 13 case presenting with a number of common and uncommon features of the syndrome. Turkish J Pediatr 2008;50:595-599.

[38] Ariel I, Wells TR, Landing BH, Singer DB. The urinary system in Down syndrome: a study of 124 autopsy cases. Pediatr Pathol 1991;11:879-888.

[39] Uehling DT, Gilbert E, Chesney R. Urologic implications of the VATER association. J Urol 1983;129:352-354.

[40] Solomon BD. VACTERL/VATER association. Orphanet J Rare Dis 2011;6:S6.

[41] Solomon BD, Baker LA, Bear KA, et al. An approach to the identification of anomalies and etiologies in neonates with identified or suspected VACTERL (Vertebral Defects, Anal Atresia, TracheoEsophageal fistula with Esophageal Atresia, Cardiac Anomalies, Renal Anomalies, and Limb Anomalies) Association. J Pediatr 2014;164:451-457.

[42] Potter EL. Bilateral renal agenesis. J Pediatr 1946;29:68-76.

[43] Humbert C, Silbermann F, Morar B, et al. Integrin alpha 8 recessive mutations are responsible for bilateral renal agenesis in humans. Am J Hum Genet 2014;94:288-294.

[44] Bruce JH, Romaguera RL, Rodriguez MM, et al. Caudal dysplasia syndrome and sirenomelia: are they part of a spectrum? Fetal Pediatr Pathol 2009;28:109-131.

[45] Herrmann J, Brauer M, Scheer I, et al. Extrahepatic biliary atresia and caudal regression syndrome in an infant of a diabetic mother. J Pediatr Surg 2004;39:E20-E22.

[46] Bruyere HJ Jr, Viseskul C, Opitz JM, et al. A fetus with upper limb amelia, "caudal regression" and Dandy Walker defect with an insulin-dependent diabetic mother. Eur J Pediatr 1980;134:139-143.

[47] Thottugal AD, Charles AK, Dickinson JE, Bower C. Caudal dysgenesis and sirenomelia-single center experience suggests common pathogenic basis. Am J Med Genet part A 2010;152A:2578-2587.

[48] Grantham JJ. Rationale for early treatment of polycystic kidney disease. Pediatr Nephrol 2014, PMID: 25022529. [Epub ahead of print].

[49] Torres VE. Vasopressin antagonists in polycystic kidney disease. Semin Nephrol 2008;28:306-317.

[50] Sweeney WE, Avner ED. Pathophysiology of childhood polycystic kidney diseases: new insights into disease-specific therapy. Pediatr Res 2014;75:148-157. 